Review

\title{
Fungal Pigments and Their Prospects in Different Industries
}

\author{
Ajay C. Lagashetti ${ }^{1}$, Laurent Dufossé ${ }^{2, * \mathbb{D}}$, Sanjay K. Singh ${ }^{1, *}$ and Paras N. Singh ${ }^{1}$ \\ 1 Biodiversity and Palaeobiology Group, National Fungal Culture Collection of India (NFCCI), \\ MACS' Agharkar Research Institute, G.G. Agarkar Road, Pune 411004, India; \\ lagashettiajay@gmail.com (A.C.L.); pnsingh@aripune.org (P.N.S.) \\ 2 Chimie et Biotechnologie des Produits Naturels \& ESIROI Agroalimentaire, Université de la Réunion, \\ 15 Avenue René Cassin, CS 92003, F-97744 Saint-Denis CEDEX, France \\ * Correspondence: laurent.dufosse@univ-reunion.fr (L.D.); sksingh@aripune.org (S.K.S.); \\ Tel.: +91-020-2532-5103 (S.K.S.)
}

Received: 17 October 2019; Accepted: 18 November 2019; Published: 22 November 2019

\begin{abstract}
The public's demand for natural, eco-friendly, and safe pigments is significantly increasing in the current era. Natural pigments, especially fungal pigments, are receiving more attention and seem to be in high demand worldwide. The immense advantages of fungal pigments over other natural or synthetic pigments have opened new avenues in the market for a wide range of applications in different industries. In addition to coloring properties, other beneficial attributes of fungal pigments, such as antimicrobial, anticancer, antioxidant, and cytotoxic activity, have expanded their use in different sectors. This review deals with the study of fungal pigments and their applications and sheds light on future prospects and challenges in the field of fungal pigments. Furthermore, the possible application of fungal pigments in the textile industry is also addressed.
\end{abstract}

Keywords: color; natural pigments; fungal pigments; dyeing; textile fabrics

\section{Introduction}

Color has always played an important role in the life of all organisms on Earth. Human life has become truly "colorful" due to the use of colors in all its aspects, including clothes, food, and furniture. Much archaeological evidence has shown that the use of pigments as coloring agents has been practiced since ancient times [1]. Pigments, especially synthetic ones, have occupied the entire market due to their wide range of applications in different industries since their discovery in the 19th century. Different attributes such as low production costs, ease of production, and superior coloring properties have largely contributed to the establishment of synthetic pigments in the market. However, the use of synthetic colors has been found to be detrimental to human health and the environment because of their many adverse impacts [2-7]. Many disadvantages of synthetic pigments, such as poor degradation, longer persistence, potential to cause cancers/allergies, etc., have increased the demand for natural, organic, and eco-friendly pigments in the current era.

The global response, as well as the demand for eco-friendly natural pigments, has significantly increased in recent decades due to their advantages over hazardous synthetic pigments. They are used as colorants, color intensifiers, additives, antioxidants, etc., in many industries including the textile, pharmaceutical, cosmetic, painting, food, and beverage industries [1,8]. In recent years, fungi have emerged among the prominent, eco-friendly sources of natural pigments. Easy processing, fast growth in cheap media, and weather-independent growth make them an excellent alternative to natural pigments. The present review highlights the role of fungi as small factories in pigment production and their potential application in different industries, including the textile industry. 


\section{Natural Pigments}

Natural pigments are naturally derived pigments synthesized mainly by plants, animals, and microbes [5,9]. Most of the natural pigments used for different purposes since ancient times are produced from plants, such as annatto, grapes, indigo, beetroot, turmeric, madder, saffron, etc. [10,11]. However, the process of pigment production from plants may not be a good option because of various problems, such as season dependency, loss of vulnerable plant species due to their extensive use, variations in color shades and intensity, expensive production, and issues related to stability and solubility [2].

Nowadays, microorganisms, including bacteria, fungi, and algae, have been shown to be an excellent alternative source of natural pigments. For the large-scale production of pigments, microorganisms are more suitable, due to a clear understanding of their cultural techniques, processing, and ease of handling. Natural pigments from microbes, especially from bacteria and fungi, have been reported worldwide by many researchers [1,10,12-20]. Many bacterial species have been reported to possess potential for pigment production [10,21-23], but their pathogenic nature as well as associated toxicity have blocked production and commercialization. This eventually opened a new avenue for producing pigments from fungi and for their various applications.

\section{Fungal Pigments}

Fungi have been shown to be a good and readily available alternative source of natural pigments $[1,20,24-26]$. Fungi have immense advantages over plants such as season-independent pigment production, easy and fast growth in a cheap culture medium, production of pigments with different color shades and of more stable, soluble pigments, and easy processing [10,27]. Fungi belonging to the Monascaceae, Trichocomaceae, Nectriaceae, Hypocreaceae, Pleosporaceae, Cordycipitaceae, Xylariaceae, Chaetomiaceae, Sordariaceae, Chlorociboriaceae, Hyaloscyphaceae, Hymenochaetaceae, Polyporaceae, Ophiostomataceae, Tremellaceae, Herpotrichiellaceae, and Tuberaceae families have been described as potent pigment producers $[8,12,20,25,26,28-45]$ (Table 1 ). These fungi are known to synthesize a variety of pigments as secondary metabolites. They are prolific producers of pigments belonging to several chemical classes, such as carotenoids, melanins, azaphilones, flavins, phenazines, quinones, monascin, violacein, indigo, etc. [16,25,26,46-49] (Table 1).

The use of Monascus pigments for the production of red mold rice (ang-kak) is the oldest recorded use of fungal pigments by humans. Certain species of Monascus, viz., Monascus ruber and Monascus purpureus, have been reported to be good potential producers of pigments worldwide. Studies have shown the potential of the red pigment produced by M. ruber as an important food colorant as well as food additive [50,51]. Many new pigments produced by $M$. ruber, such as $N$-glucosylrubropunctamine, $\mathrm{N}$-glucosylmonascorubramine, monarubrin, rubropunctin, etc., have been discovered (Figure 1) [52-54]. Recently, researchers revealed the first detailed biosynthetic pathway of Monascus azophilone pigments (MonAzPs) in M. ruber M7, based on targeted gene knockouts, heterologous gene expression, as well as in vitro enzymatic and chemical reactions [55]. Along with M. ruber, M. purpureus was also reported to produce a variety of novel pigments, such as monapurone $\mathrm{A}-\mathrm{C}$, monasphilone $\mathrm{A}-\mathrm{B}$, monapilol A-D, and 9-(1-hydroxyhexyl)-3-(2-hydroxypropyl)-6a-methyl-9,9a-dihydrofuro[2,3-h] isoquinoline-6,8 $(2 \mathrm{H}, 6 \mathrm{aH})$-dione (Figure 1) [56-59]. Another study reports on the physicochemical $(\mathrm{pH}$, light, and heat stability) properties of the red pigment of M. purpureus [60]. 
Table 1. Updated list of pigment-producing fungi and their respective pigments [25,61].

\begin{tabular}{|c|c|c|}
\hline Fungal Species & Pigments & References \\
\hline \multicolumn{3}{|c|}{ Monascus species } \\
\hline Monascus pilosus & Citrinin (yellow) & [61] \\
\hline Monascus purpureus & $\begin{array}{l}\text { Monascin (yellow), monascorubrin (orange), monascorubramine (red), } \\
\text { monapurone A-C (yellow), monasphilone A and B (yellow), ankaflavin } \\
\text { (yellow), rubropunctamine (purple-red), rubropunctatin (orange), } \\
\text { monopilol A-D (yellow), citrinin (yellow), } \\
\text { 9-(1-hydroxyhexyl)-3-(2-hydroxypropyl)-6a-methyl-9,9a-dihydrofuro[2,3-h] } \\
\text { isoquinoline-6,8(2H,6aH)-dione (red), uncharacterized (red) }\end{array}$ & [56-61] \\
\hline Monascus ruber & $\begin{array}{l}\text { Monascin (yellow), monascorubramine (red), monascorubrin (orange), } \\
\text { ankaflavin (yellow), citrinin (yellow), rubropunctamine (purple-red), } \\
\text { rubropunctatin (orange), } N \text {-glucosylrubropunctamine (red), } \\
N \text {-glucosylmonascorubramine (red), monarubrin (pale yellow), } \\
\text { rubropunctin (pale yellow) }\end{array}$ & {$[52,54,61]$} \\
\hline Monascus species & Ankaflavin (yellow) ${ }^{*}$, monascorubramine $(\text { red })^{*}$, rubropunctatin (orange) ${ }^{*}$ & [25] \\
\hline \multicolumn{3}{|c|}{ Fusarium species } \\
\hline $\begin{array}{l}\text { Fusarium acuminatum, } \\
\text { F. avenaceum, F. tricinctum }\end{array}$ & Antibiotic Y (yellow), aurofusarin (red) & [61] \\
\hline Fusarium chlamydosporum & Uncharacterized (red) & [62] \\
\hline Fusarium culmorum & Aurofusarin (red), fuscofusarin (yellow), rubrofusarin (red) & [61] \\
\hline $\begin{array}{l}\text { Fusarium fujikuroi (formerly } \\
\text { known as Fusarium } \\
\text { moniliforme/ Fusarium } \\
\text { verticillioides) }\end{array}$ & $\begin{array}{l}\text { Bikaverin (red), norbikaverin (red), } O \text {-demethylanhydrofusarubin (red), } \\
\text { 8-O-methybostrycoidin, 2-(4-((3E,5E)-14-aminotetradeca-3,5-dienyloxy) } \\
\text { butyl)-1,2,3,4-tetrahydroisoquinolin-4-ol (ATDBTHIQN) (pink), } \\
\text { neurosporaxanthin (orange), } \beta \text {-carotene (red-orange), fusarubin (red), } \\
O \text {-demethylfusarubin, } O \text {-methyljavanicin, } O \text {-methylsolaniol (orange-red) }\end{array}$ & {$[43,61,63-65]$} \\
\hline Fusarium graminearum & $\begin{array}{l}\text { Aurofusarin (red,) rubrofusarin (red), 5-deoxybostrycoidin anthrone } \\
\text { (green), 6-O-demethyl-5-deoxybostrycoidin anthrone (blue), } \\
\text { purpurfusarin (purple), 6-O-demethyl-5-deoxybostrycoidin (yellow), } \\
\text { 5-deoxybostrycoidin (red) }\end{array}$ & {$[64,66]$} \\
\hline Fusarium oxysporum & $\begin{array}{l}\text { 2,7-dimethoxy-6-(acetoxyethyl)juglone (yellow), bikaverin (red), } \\
\text { bostrycoidin (red), nectriafurone (yellow), norjavanicin (red), O-methyl-6- } \\
\text { hydroxynorjavanicin (yellow), O-methylanhydrofusarubin (orange-red), } \\
\text { O-methylfusarubin (red), O-methyljavanicin, } \\
\text { 2-acetyl-3,8-dihydroxy-6-methoxy anthraquinone (yellow), } \\
\text { 2-(1-hydroxyethyl)-3,8-dihydroxy-6-methoxy anthraquinone (orange), } \\
\text { neurosporaxanthin (orange), } \beta \text {-carotene (red-orange), uncharacterized } \\
\text { naphthaquinones (purple) }\end{array}$ & {$[43,47,61,64,67]$} \\
\hline $\begin{array}{l}\text { Fusarium poae, } \\
\text { F. sambucinum }\end{array}$ & Aurofusarin (red) & \multirow[t]{2}{*}{ [61] } \\
\hline Fusarium solani & $\begin{array}{l}\text { Fusarubin (red), O-methyldihydrofusarubin (red), O-ethylfusarubin (red), } \\
\text { isomarticins (red) }\end{array}$ & \\
\hline Fusarium sporotrichioides & Aurofusarin (red), $\beta$-carotene (yellow-orange) ${ }^{* *}$, lycopene (red) ${ }^{* *}$ & {$[25,61]$} \\
\hline Fusarium stilboides & Antibiotic Y (yellow), aurofusarin (red), nectriafurone (yellow) & \multirow{2}{*}{ [61] } \\
\hline Fusarium venenatum & Aurofusarin (red), rubrofusarin (red) & \\
\hline Fusarium sp. & Benzoquinone (yellow) & [68] \\
\hline $\begin{array}{l}\text { Fusarium sp. PSU-F14 and } \\
\text { PSU-F135 }\end{array}$ & Fusarnaphthoquinones B (red), fusarnaphthoquinones C (red) & [69] \\
\hline \multicolumn{3}{|c|}{ Fusicolla aquaeductuum (Formerly Known as Fusarium aquaeductuum) } \\
\hline Fusicolla aquaeductuum & Neurosporaxanthin (orange), $\beta$-carotene (red-orange) & [43] \\
\hline \multicolumn{3}{|c|}{ Albonectria rigidiuscula (Formerly Known as Fusarium decemcellulare) } \\
\hline Albonectria rigidiuscula & $\begin{array}{l}\text { Javanicin (red-orange), fusarubin (red), anhydrojavanicin, } \\
\text { anhydrofusarubin, bostricoidin (red), novarubin }\end{array}$ & [64] \\
\hline
\end{tabular}


Table 1. Cont.

\begin{tabular}{|c|c|c|}
\hline Fungal Species & Pigments & References \\
\hline \multicolumn{3}{|c|}{ Trichoderma species } \\
\hline Trichoderma harzianum & $\begin{array}{l}\text { Pachybasin (yellow), chrysophanol (orange-red), emodin (yellow), } \\
\text { 1-hydroxy-3-methyl-anthraquinone, } \\
\text { 1,8-dihydroxy-3-methyl-anthraquinone, T22 azaphilone }\end{array}$ & \multirow{4}{*}{ [25] } \\
\hline Trichoderma polysporum & Pachybasin (yellow), chrysophanol (orange-red), emodin (yellow) & \\
\hline Trichoderma viride & $\begin{array}{l}\text { Pachybasin (yellow), chrysophanol (orange-red), emodin (yellow), } \\
\text { 1,3,6,8-tetrahydroxyanthraquinone, 2,4,5,7- tetrahydroxyanthraquinone }\end{array}$ & \\
\hline Trichoderma aureoviride & Pachybasin (yellow), chrysophanol (orange-red) & \\
\hline $\begin{array}{l}\text { Trichoderma afrharzianum, } \\
\text { Trichoderma pyramidale, } \\
\text { Trichoderma parareesei } \\
\text { (formerly known as } \\
\text { Trichoderma atroviride), } \\
\text { Trichoderma sp. } 1\end{array}$ & Uncharacterized (yellow) & {$[70,71]$} \\
\hline Trichoderma parceramosum & Uncharacterized (red) & [72] \\
\hline \multicolumn{3}{|c|}{ Cordyceps farinosa (Formerly Known as Isaria farinosa) } \\
\hline Cordyceps farinosa & Anthraquinone derivative & [73] \\
\hline \multicolumn{3}{|c|}{ Ophiocordyceps unilateralis (Formerly Known as Cordyceps unilateralis) } \\
\hline Ophiocordyceps unilateralis & $\begin{array}{l}\text { Erythrostominone (red), 3,5,8-TMON * (red), deoxyerythrostominone (red), } \\
\text { deoxyerythrostominol (red), 4-O-methyl erythrostominone (red), } \\
\text { epierythrostominol (red), naphthoquinones (deep blood red) }\end{array}$ & [25] \\
\hline \multicolumn{3}{|c|}{ Beauveria species } \\
\hline Beauveria basiana & $\begin{array}{l}\text { Tenellin (yellow), bassianin (yellow), pyridovericin (pale yellow), } \\
\text { pyridomacrolidin (pale yellow), oosporein (red) }\end{array}$ & \multirow[b]{2}{*}[25,74]{} \\
\hline $\begin{array}{l}\text { Beauveria brongniartii } \\
\text { (formerly known as } \\
\text { Beauveria tenella) }\end{array}$ & Tenellin (yellow), bassianin (yellow) & \\
\hline \multicolumn{3}{|c|}{ Torrubiella species } \\
\hline Torrubiella sp. & Torrubiellones A-D (yellow) & [75] \\
\hline \multicolumn{3}{|c|}{ Lecanicillium species } \\
\hline Lecanicillium aphanocladii & Oosporein (red) & [41] \\
\hline \multicolumn{3}{|c|}{ Hyperdermium species } \\
\hline Hyperdermium bertonii & Skyrin (orange-red) & [25] \\
\hline \multicolumn{3}{|c|}{ Daldinia species } \\
\hline $\begin{array}{l}\text { Daldinia bambusicol, } \\
\text { Daldinia caldariorum, } \\
\text { Daldinia childiae, } \\
\text { Daldinia clavata, } \\
\text { Daldinia fissa, } \\
\text { Daldinia grandis, } \\
\text { Daldinia lloydi, } \\
\text { Daldinia loculata, } \\
\text { Daldinia petriniae, } \\
\text { Daldinia singularis }\end{array}$ & $\begin{array}{l}\text { BNT }\left(1,1^{\prime} \text {-Binaphthalene-4,4'-5,5'-tetrol) (yellow), daldinol (dark brown), }\right. \\
8 \text {-methoxy-1-napthol, 2-hydroxy-5-methylchromone }\end{array}$ & [25] \\
\hline Daldinia concentrica & $\begin{array}{l}\text { BNT }\left(1,1^{\prime} \text {-Binaphthalene- } 4,4^{\prime}-5,5^{\prime} \text {-tetrol) (yellow), daldinol, }\right. \\
\text { 8-methoxy-1-napthol, 2-hydroxy-5-methylchromone, daldinal A-C } \\
\text { (yellow), daldinin A-C (green-olivaceous-isabelline) }\end{array}$ & \\
\hline Daldinia eschscholzii & $\begin{array}{l}\text { BNT (1,1'-Binaphthalene-4,4'-5,5'-tetrol) (yellow), daldiol (dark brown), } \\
\text { 8-methoxy-1-napthol, 2-hydroxy-5-methylchromone, daldinal A-C (yellow) }\end{array}$ & \\
\hline
\end{tabular}


Table 1. Cont.

\begin{tabular}{|c|c|c|}
\hline Fungal Species & Pigments & References \\
\hline \multicolumn{3}{|c|}{ Jackrogersella cohaerens (Formerly Known as Annulohypoxylon cohaerens) } \\
\hline Jackrogersella cohaerens & Cohaerin A & [25] \\
\hline \multicolumn{3}{|c|}{ Hypoxylon species } \\
\hline Hypoxylon fragiforme & $\begin{array}{l}\text { Hypoxyxylerone (green), fragiformins A-B, cytochalasin H (white), } \\
\text { mitorubrin azaphilones (red) }\end{array}$ & \multirow{9}{*}{ [25] } \\
\hline Hypoxylon howeanum & Mitorubrin azaphilones (red) & \\
\hline Hypoxylon lechatii & Vermelhotin (orange-red), hypoxyvermelhotins A-C (orange-red) & \\
\hline Hypoxylon fuscum & Daldinin A-C (green-olivaceous-isabelline) & \\
\hline $\begin{array}{l}\text { Hypoxylon } \\
\text { fulvo-sulphureum }\end{array}$ & Mitorubrinol derivatives & \\
\hline Hypoxylon sclerophaeum & Hypoxylone (orange) & \\
\hline Hypoxylon rickii & Rickenyl B (red), rickenyl D (brown) & \\
\hline $\begin{array}{l}\text { Hypoxylon lenormandii, } \\
\text { Hypoxylon jaklitschii }\end{array}$ & Lenormandins A-G (yellow) & \\
\hline Hypoxylon rubiginosum & $\begin{array}{l}\text { Mitorubrin (orange), rubiginosin (orange-brown), hypomiltin } \\
\text { (yellowish-green) }\end{array}$ & \\
\hline \multicolumn{3}{|c|}{ Alternaria species } \\
\hline Alternaria alternata & $\begin{array}{l}\text { Alternariol (red), altenuene (red-violet), alternarienoic acid (red), } \\
\text { alternariol-5-methyl ether (red-brown), tenuazoic acid (orange-red), } \\
\text { alterperylenol (red), stemphyperylenol (yellow-orange-red) }\end{array}$ & [76] \\
\hline Aternaria dauci & Uncharacterized (red) & {$[25,61]$} \\
\hline Aternaria porri & Altersolanol A (yellow-orange), dactylariol & {$[25,61,77]$} \\
\hline $\begin{array}{l}\text { Aternaria solani, Aternaria } \\
\text { tomatophila }\end{array}$ & Altersolanol A (yellow-orange) & {$[25,61]$} \\
\hline Alternaria species & Alterperylenol (red), dihydroalterperylenol (dark purple) & [78] \\
\hline Alternaria sp. ZJ9-6B & Alterporriol K-M (red) & [79] \\
\hline \multicolumn{3}{|c|}{ Curvularia species } \\
\hline Curvularia lunata & $\begin{array}{l}\text { Chrysophanol (red), cynodontin (bronze), helminthosporin (maroon), } \\
\text { erythroglaucin (red), catenarin (red) }\end{array}$ & {$[25,61]$} \\
\hline \multicolumn{3}{|c|}{ Sanghuangporus species } \\
\hline Sanghuangporus baumii & Uncharacterized (yellow) & [71] \\
\hline \multicolumn{3}{|c|}{ Clonostachys species } \\
\hline Clonostachys intermedia & Uncharacterized (yellow) & [71] \\
\hline \multicolumn{3}{|c|}{ Pyrenophora species (Previously Known as species of Drechslera) } \\
\hline $\begin{array}{l}\text { Pyrenophora teres, } \\
\text { Pyrenophora graminea, } \\
\text { Pyrenophora tritici-repentis, } \\
\text { Pyrenophora grahamii, } \\
\text { Pyrenophora dictyoides, } \\
\text { Pyrenophora chaetomioides }\end{array}$ & $\begin{array}{l}\text { Catenarin (red), cynodontin (bronze), helminthosporin (maroon), tritisporin } \\
\text { (reddish-brown), erythroglaucin (red) }\end{array}$ & {$[25,61]$} \\
\hline \multicolumn{3}{|c|}{ Exophiala species } \\
\hline $\begin{array}{l}\text { Exophiala dermatitidis } \\
\text { (formerly known as } \\
\text { Wangiella dermatitidis) }\end{array}$ & Melanin (black-brown) & [44] \\
\hline \multicolumn{3}{|c|}{ Sporothrix species } \\
\hline Sporothrix schenckii & Melanin (black-brown) & [44] \\
\hline
\end{tabular}


Table 1. Cont.

\begin{tabular}{|c|c|c|}
\hline Fungal Species & Pigments & References \\
\hline \multicolumn{3}{|c|}{ Cryptococcus species } \\
\hline Cryptococcus neoformans & Dihydroxy phenyl alanine-melanin & {$[29,80]$} \\
\hline \multicolumn{3}{|c|}{ Tuber species } \\
\hline Tuber melanosporum & Melanin (black) & {$[29,81]$} \\
\hline \multicolumn{3}{|c|}{ Polyporus species } \\
\hline $\begin{array}{l}\text { Lentinus brumalis } \\
\text { (formerly known as } \\
\text { Polyporus brumalis) }\end{array}$ & Melanin (black) & \multirow[t]{2}{*}[34,35]{} \\
\hline $\begin{array}{l}\text { Cerioporus squamosus } \\
\text { (formerly known as } \\
\text { Polyporus squamosus) }\end{array}$ & Melanin (black) & \\
\hline \multicolumn{3}{|c|}{ Xylaria species } \\
\hline Xylaria polymorpha & Melanin (black) & {$[34,35]$} \\
\hline \multicolumn{3}{|c|}{ Fomes species } \\
\hline Fomes fomentarius & Melanin (black) & {$[34,35]$} \\
\hline \multicolumn{3}{|c|}{ Oxyporus species } \\
\hline Oxyporus populinus & Melanin (black) & [34] \\
\hline \multicolumn{3}{|c|}{ Trametes species } \\
\hline Trametes versicolor & Melanin (black) & {$[34,35]$} \\
\hline \multicolumn{3}{|c|}{ Inonotus species } \\
\hline Inonotus hispidus & Melanin (black), uncharacterized (yellow) & [34-36] \\
\hline \multicolumn{3}{|c|}{ Chlorociboria species } \\
\hline Chlorociboria aeruginascens & Xylindein (green), xylindein quinol (yellow) & [33] \\
\hline Chlorociboria aeruginosa & Xylindein (green) & {$[37,39]$} \\
\hline \multicolumn{3}{|c|}{ Scytalidium species } \\
\hline Scytalidium cuboideum & Draconin red (red) & {$[37,39]$} \\
\hline $\begin{array}{l}\text { Scytalidium } \\
\text { ganodermophthorum }\end{array}$ & Uncharacterized (yellow) & {$[36,39]$} \\
\hline Scytalidium lignicola & Uncharacterized (yellow) & {$[36,39]$} \\
\hline \multicolumn{3}{|c|}{ Epicoccum species } \\
\hline Epicoccum nigrum & $\begin{array}{l}\text { Carotenoids, chromanone (yellow), epicoccarines A-B, epicocconone } \\
\text { (fluorescent yellow), epipyridone (red), flavipin (brown), isobenzofuran } \\
\text { derivatives (yellow to brown), orevactaene (yellow) }\end{array}$ & {$[41,61]$} \\
\hline \multicolumn{3}{|c|}{ Chaetomium species } \\
\hline Chaetomium cupreum & Oosporein (red), rotiorinols A-C (red), rubrorotiorin (red) & [25] \\
\hline Chaetomium globosum & $\begin{array}{l}\text { Chaetoviridins A-D (yellow), chaetoglobin A-B, chaetomugilins A-F, } \\
\text { cochliodinol (purple) }\end{array}$ & \\
\hline $\begin{array}{l}\text { Chaetomium sp. } \\
\text { NA-S01-R1 }\end{array}$ & Chaephilone-C (yellow), chaetoviridides A-C (red) & [82] \\
\hline \multicolumn{3}{|c|}{ Achaetomium species } \\
\hline Achaetomium sp. & Parietin (orange) & [25] \\
\hline \multicolumn{3}{|c|}{ Phyllosticta species } \\
\hline Phyllosticta capitalensis & Melanin (black) & [83] \\
\hline \multicolumn{3}{|c|}{ Cladosporium species } \\
\hline Cladosporium cladosporioides & Calphostins A-D and I (red) & [61] \\
\hline
\end{tabular}


Table 1. Cont

\begin{tabular}{|c|c|c|}
\hline Fungal Species & Pigments & References \\
\hline \multicolumn{3}{|c|}{ Nodulisporium species } \\
\hline Nodulisporium hinnuleum & Hinnuliquinone (red) & [84] \\
\hline \multicolumn{3}{|c|}{ Astrosphaeriella species } \\
\hline Astrosphaeriella papuana & Astropaquinones A-C (orange) & [85] \\
\hline \multicolumn{3}{|c|}{ Arthrobotrys species } \\
\hline Arthrobotrys ferox & Carotenoid & [86] \\
\hline \multicolumn{3}{|c|}{ Thelebolus species } \\
\hline Thelebolus microsporus & $\beta$-carotene (orange) & {$[86,87]$} \\
\hline \multicolumn{3}{|c|}{ Shiraia species } \\
\hline Shiraia bambusicola & Shiraiarin (red), hypocrellin D (orange-red) & {$[88,89]$} \\
\hline \multicolumn{3}{|c|}{ Paecilomyces species } \\
\hline Paecilomyces sinclairii & Uncharacterized (red) $* *$ & {$[25,61]$} \\
\hline \multicolumn{3}{|c|}{ Neurospora species } \\
\hline Neurospora crassa & $\begin{array}{l}\text { Neurosporaxanthin (yellow-orange), phytoene (yellow-orange), } \beta \text {-carotene } \\
\text { (red-orange), lycopene (red), neurosporen (yellow-orange), spirilloxanthin } \\
\text { (violet), Y-carotene (yellow-orange), } \beta \text {-carotene (yellow-orange) }\end{array}$ & {$[25,90]$} \\
\hline Neurospora sitophila & Neurosporaxanthin (yellow-orange) & \multirow{2}{*}{ [26] } \\
\hline Neurospora intermedia & Uncharacterized (yellow-orange), a mixture of carotenoids & \\
\hline \multicolumn{3}{|c|}{ Blakeslea species } \\
\hline Blakeslea trispora & $\beta$-carotene (yellow-orange) ${ }^{*}$, lycopene (red) ${ }^{*}$ & [25] \\
\hline \multicolumn{3}{|c|}{ Ashbya species } \\
\hline Ashbya gossypi & Riboflavin (yellow) * & [25] \\
\hline \multicolumn{3}{|c|}{ Phycomyces species } \\
\hline Phycomyces blakesleeanus & $\beta$-carotene (yellow-orange) ** & [25] \\
\hline \multicolumn{3}{|c|}{ Mucor species } \\
\hline Mucor circinelloides & $\beta$-carotene (yellow-orange) ${ }^{* * *}$ & [25] \\
\hline \multicolumn{3}{|c|}{ Lactarius species } \\
\hline Lactarius sp. & Azulenes (blue) ${ }^{* *}$ & [25] \\
\hline \multicolumn{3}{|c|}{ Penicillium species } \\
\hline Penicillium atramentosum & Uncharacterized (dark brown) & \multirow{5}{*}[61,91]{} \\
\hline Penicillium atrosanguineum & Phoenicin (red), uncharacterized (yellow and red) & \\
\hline Penicillium atrovenetum & Atrovenetin (yellow), norherqueinone (red) & \\
\hline Penicillium aurantiogriseum & Uncharacterized & \\
\hline $\begin{array}{l}\text { Penicillium brevicompactum, } \\
\text { Penicillium simplicissimum }\end{array}$ & Xanthoepocin (yellow) & \\
\hline Penicillium chrysogenum & Sorbicillins (yellow), xanthocillin (yellow), chrysogine (yellow) & {$[61,92]$} \\
\hline Penicillium citrinum & Anthraquinones (yellow), citrinin (yellow) & [61] \\
\hline $\begin{array}{l}\text { Penicillium convolutum } \\
\text { (formerly known as } \\
\text { Talaromyces convolutus) }\end{array}$ & Talaroconvolutins A-D, ZG-1494 $\alpha$ & [93] \\
\hline Penicillium cyclopium & Viomellein (reddish-brown), xanthomegnin (orange) & \multirow{3}{*}{ [61] } \\
\hline Penicillium discolor & Uncharacterized & \\
\hline Penicillium echinulatum & Uncharacterized (yellow) & \\
\hline Penicillium flavigenum & Xanthocillin (yellow), dihydrotrichodimerol (yellow) & {$[41,61]$} \\
\hline
\end{tabular}


Table 1. Cont.

\begin{tabular}{|c|c|c|}
\hline Fungal Species & Pigments & References \\
\hline \multicolumn{3}{|c|}{ Penicillium species } \\
\hline $\begin{array}{l}\text { Penicillium freii, Penicillium } \\
\text { viridicatum }\end{array}$ & Viomellein (reddish-brown), vioxanthin, xanthomegnin (orange) & [61] \\
\hline Penicillium herquei & Atrovenetin (yellow), herqueinones (red and yellow) & \\
\hline Penicillium melinii & Atrovenetin (yellow) & [91] \\
\hline Penicillium miczynskii & Uncharacterized (red) & [71] \\
\hline Penicillium mallochii & Sclerotiorin (yellow) & [94] \\
\hline Penicillium oxalicum & $\begin{array}{l}\text { Arpink red }{ }^{\mathrm{TM}} \text {, anthraquinone derivative (red), secalonic acid D (yellow), } \\
\text { anthraquinones (red and other hues) * }\end{array}$ & {$[25,61]$} \\
\hline Penicillium paneum & Uncharacterized (red) & [61] \\
\hline Penicillium persicinum & Uncharacterized (cherry red) & \\
\hline Penicillium sp. AZ & PP-V (violet), PP-R (red) & [95] \\
\hline Penicillium sp. (GBPI_P155) & Uncharacterized (orange) & [96] \\
\hline Penicillium sp. NIOM-02 & Uncharacterized (red) & [97] \\
\hline Penicillium sp. & Uncharacterized (red) & {$[98,99]$} \\
\hline \multicolumn{3}{|c|}{ Talaromyces species } \\
\hline $\begin{array}{l}\text { Talaromyces aculeatus } \\
\text { (formerly known as } \\
\text { Penicillium aculeatum) }\end{array}$ & Uncharacterized & [61] \\
\hline Talaromyces atroroseus & $\begin{array}{l}\text { Mitorubrin (red), monascorubrin (red), PP-R (red), glauconic acid (red), } \\
\text { purpuride (red), ZG-1494 } \alpha \text { (red), azaphilones (red)*** }\end{array}$ & {$[25,100]$} \\
\hline $\begin{array}{l}\text { Talaromyces albobiverticillius, } \\
\text { Talaromyces amestolkiae, } \\
\text { Talaromyces stollii }\end{array}$ & Monascus-like azaphilones (red) & [25] \\
\hline $\begin{array}{l}\text { Talaromyces cnidii, } \\
\text { Talaromyces coalescens }\end{array}$ & Monascus-like azaphilones (red), uncharacterized (red) & \\
\hline $\begin{array}{l}\text { Talaromyces funiculosus } \\
\text { (formerly known as } \\
\text { Penicillium funiculosum) }\end{array}$ & Ankaflavain (yellow), uncharacterized & [61] \\
\hline $\begin{array}{l}\text { Talaromyces islandicus } \\
\text { (formerly known as } \\
\text { Penicillium islandicum) }\end{array}$ & $\begin{array}{l}\text { Emodin (yellow), skyrin (orange), erythroskyrin (orange-red), luteoskyrin } \\
\text { (yellow) }\end{array}$ & \\
\hline $\begin{array}{l}\text { Talaromyces marneffei } \\
\text { (formerly known as } \\
\text { Penicillium marneffiei) }\end{array}$ & $\begin{array}{l}\text { Monascorubramine (purple-red), mitorubrinol (orange-red), rubropunctatin } \\
\text { (orange), purpactin, herqueinone like (brick red), secalonic acid D (yellow) }\end{array}$ & {$[61,101]$} \\
\hline $\begin{array}{l}\text { Talaromyces pinophilus } \\
\text { (formerly known as } \\
\text { Penicillium pinophilum) }\end{array}$ & Azaphilones, uncharacterized & {$[25,61]$} \\
\hline $\begin{array}{l}\text { Talaromyces purpureogenus } \\
\text { (formerly known as } \\
\text { Penicillium purpureogenum) }\end{array}$ & $\begin{array}{l}\text { Mitorubrin (yellow), mitorubrinol (orange-red), PP-R (purple-red), } \\
\text { purpurogenone (yellow-orange), rubropunctatin (red), } \\
N \text {-glutarylmonascorubramine, } N \text {-glutarylrubropunctamine, } \\
\text { uncharacterized (red), azaphilones (red) }\end{array}$ & {$[25,61,102-105]$} \\
\hline $\begin{array}{l}\text { Talaromyces ruber (formerly } \\
\text { known as Penicillium } \\
\text { crateriforme) }\end{array}$ & Uncharacterized, Monascus-like azaphilones & [25] \\
\hline $\begin{array}{l}\text { Talaromyces rugulosus } \\
\text { (formerly known as } \\
\text { Penicillium rugulosum) }\end{array}$ & Rugulosin (yellow) & [61] \\
\hline $\begin{array}{l}\text { Talaromyces variabillis } \\
\text { (formerly known as } \\
\text { Penicillium variabile) }\end{array}$ & Rugulosin (yellow) & [61] \\
\hline
\end{tabular}


Table 1. Cont.

\begin{tabular}{|c|c|c|}
\hline Fungal Species & Pigments & References \\
\hline Talaromyces vericulosus & Uncharacterized (red) & [106] \\
\hline Talaromyces sp. DgCr22.1b & Talaroxanthone (yellow) & [107] \\
\hline $\begin{array}{l}\text { Talaromyces siamensis, } \\
\text { Talaromyces sp. }\end{array}$ & Uncharacterized (red) & {$[71,108]$} \\
\hline Talaromyces sp. & $\mathrm{N}$-threonine rubropunctamine (red) & [72] \\
\hline \multicolumn{3}{|c|}{ Hamigera avellanea (Formerly Known as Talaromyces avellaneus) } \\
\hline Hamigera avellanea & Emodin (yellow), erythroglaucin (red), catenarin (red) & [109] \\
\hline \multicolumn{3}{|c|}{ Aspergillus species } \\
\hline Aspergillus amstelodami & $\begin{array}{l}\text { Physcion (yellow), erythroglaucin (red), flavoglaucin (yellow), auroglaucin } \\
\text { (orange-red) }\end{array}$ & [25] \\
\hline Aspergillus awamori & Asperenone (yellow) & [110] \\
\hline Aspergillus chevalieri & $\begin{array}{l}\text { Physcion (yellow), erythroglaucin (red), flavoglaucin (yellow), auroglaucin } \\
\text { (orange-red), catenarin (red), rubrocristin (red) }\end{array}$ & [25] \\
\hline Aspergillus cristatus & $\begin{array}{l}\text { Emodin (yellow), questin (yellow to orange-brown), erythroglaucin (red), } \\
\text { physcion (yellow), catenarin (red), rubrocristin (red) }\end{array}$ & {$[25,61]$} \\
\hline
\end{tabular}

Aspergillus echinulatum,

Aspergillus glaber,

Aspergillus spiculosus,

Aspergillus umbrosus

\begin{tabular}{lll}
\hline Aspergillus fumigatus & Melanin (dark brown-black) \\
\hline $\begin{array}{l}\text { Aspergillus falconensis, } \\
\text { Aspergillus fruticulosus }\end{array}$ & Falconensins A-H (yellow), falconensones A1 and B2 (yellow), zeorin \\
\hline
\end{tabular}

Physcion (yellow), emodin (yellow), questin (yellow to orange-brown),

Aspergillus glaucus $\quad$ erythroglaucin (red), catenarin (red), rubrocristin (red), flavoglaucin
(yellow), auroglaucin (orange-red), aspergin (yellow)

Aspergillus intermedius,

Aspergillus leucocarpus, Physcion (yellow), erythroglaucin (red)

Aspergillus tonophilus

Aspergillus ochraceus Viomellein (reddish-brown), vioxanthin, xanthomegnin (orange)

Aspergillus melleus,

Aspergillus sulphureus,

Aspergillus westerdijkiae

Viomellein (reddish-brown), rubrosulphin (red), viopurpurin (purple),

xanthomegnin (orange)

Aspergillus nidulans Ascoquinone A (red), norsolorinic acid, sterigmatocystin (yellow), melanin (dark brown-black)

$[25,112,113]$

Aspergillus niger Flavioline (orange-red), $N$-naptho- $\gamma$-pyrones (yellow), aspergillin (black),

Aspergillus niger azanigerones A-F, asperenone (yellow), melanin (dark brown-black)

$[25,61,110,114,115]$

Aspergillus nishimurae

Aspergillus parvathecia,

Aspergillus rugulosus,

Aspergillus versicolor

Anishidiol (yellow)

Aspergillus purpureus

Sterigmatocystin (yellow)

Aspergillus repens

Epurpurins A-C (yellow)

Emodin (yellow), physcion (yellow), erythroglaucin (red), catenarin (red),

rubrocristin (red), questin (yellow to orange-brown)

Catenarin (red), rubrocristin (red), emodin (orange), asperflavin (yellow),

eurorubrin (Brown), questin (yellow to orange-brown),

Aspergillus ruber

3-O-( $\alpha-\mathrm{D}-$ ribofuranosyl)-questin (orange),

2-O-methyl-9-dehydroxyeurotinone,

2-O-methyl-4-O-( $\alpha-$ D-ribofuranosyl)-9-dehydroxyeurotinone,

2-O-methyleurotinone

\begin{tabular}{llll}
\hline Aspergillus sclerotioniger & Uncharacterized (yellow) & [61] & [91] \\
\hline Aspergillus sclerotiorum & Neoaspergillic acid (yellow-green) & [118] & [119] \\
\hline Aspergillus terreus & Uncharacterized (yellow) & [120] \\
\hline Aspergillus sp. & Ferriaspergillin (red), ferrineoaspergillin (red) & \\
\hline Aspergillus sp. & Uncharacterized (yellow) & \\
\hline
\end{tabular}

${ }^{*}$ Industrial production (IP), ${ }^{* *}$ research project (RP), ${ }^{* * *}$ development stage (DS). 
<smiles>[R]C(=O)C1C(=O)OC2(C)C(=O)C3=C(C=C(/C=C/C)OC3)CC12</smiles>

Monascin $\left(\mathrm{R}=-\mathrm{C}_{5} \mathrm{H}_{11}\right)$

Ankaflavin $\left(\mathrm{R}=-\mathrm{C}_{7} \mathrm{H}_{15}\right)$<smiles>[R]C(=O)C1=C2C=C3C=C(C=CC)OC=C3[C@@H](O)[C@@]2(C)OC1=O</smiles>

$$
\text { Monophilol A }\left(\mathrm{R}=-\mathrm{C}_{7} \mathrm{H}_{15}\right)
$$$$
\text { Monophilol B }\left(\mathrm{R}=-\mathrm{C}_{5} \mathrm{H}_{11}\right)
$$

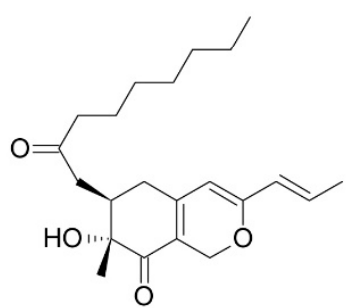

Monasphilone A<smiles>[R]C(=O)C1=C2C=C3C=C(C=CC)OC=C3C(=O)C2(C)OC1=O</smiles>

Rubropunctatin $\left(\mathrm{R}=-\mathrm{C}_{5} \mathrm{H}_{11}\right)$

Monascorubrin $\left(\mathrm{R}=-\mathrm{C}_{7} \mathrm{H}_{15}\right)$<smiles>[R]C(=O)C1=C2C=C3C=C(/C=C/C)OC=C3[C@@](C)(CC(C)=O)[C@@]2(C)OC1=O</smiles>

Monophilol C $\left(\mathrm{R}=-\mathrm{C}_{7} \mathrm{H}_{15}\right)$

Monophilol D $\left(\mathrm{R}=-\mathrm{C}_{5} \mathrm{H}_{11}\right)$<smiles>[R]C(=O)C1=C2C=C3C=C(C=CC)NC=C3C(=O)C2(C)OC1=O</smiles>

Rubropunctamine $\left(\mathrm{R}=-\mathrm{C}_{5} \mathrm{H}_{11}\right)$

Monascorubramine $\left(\mathrm{R}=-\mathrm{C}_{7} \mathrm{H}_{15}\right)$<smiles>[R]C(=O)C[C@H]1C=C2C=C(/C=C/C)OC=C2C(=O)[C@]1(C)O</smiles>

Monarubrin $\left(\mathrm{R}=-\mathrm{C}_{5} \mathrm{H}_{11}\right)$

Rubropunctin $\left(\mathrm{R}=-\mathrm{C}_{7} \mathrm{H}_{15}\right)$<smiles>C/C=C/C1=CC2=C(CO1)C(=O)C(C)(O)[C@@H](CC(=O)CCCCC)C2</smiles>

Monasphilone B

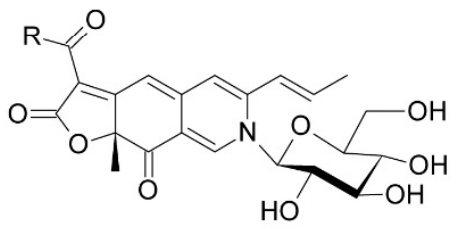

$N$-glucosylrubropunctamine $\left(\mathrm{R}=-\mathrm{C}_{5} \mathrm{H}_{11}\right)$ $\mathrm{N}$-glucosylmonascorubramine $\left(\mathrm{R}=-\mathrm{C}_{7} \mathrm{H}_{15}\right)$

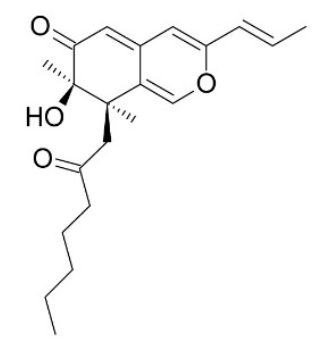

Monapurone A

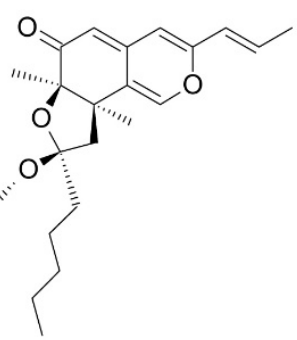

Monapurone B

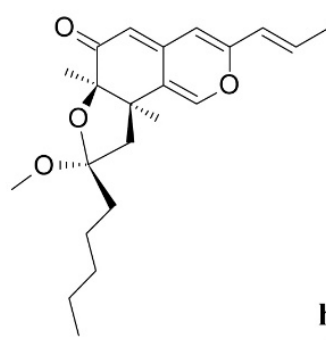

Monapurone C

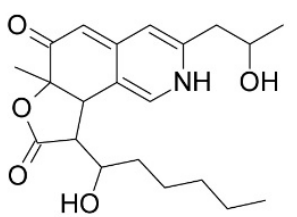

9-(1-hydroxyhexyl)-3-(2hydroxypropyl)-6a-methyl-9,9adihydrofuro[2,3-h]isoquinoline$6,8(2 \mathrm{H}, 6 \mathrm{aH})$-dione

Figure 1. Pigments reported from Monascus species (M. ruber and M. purpureus), re-drawn from [52,54,56-59].

Along with Monascus, many species of Fusarium have been reported for their capability to produce pigments. Studies have reported pigments such as bikaverin, nor-bikaverin, fusarubins, some naphthoquinone (8-O-methybostrycoidin, 8-O-methylfusarubin, 8-O-methylnectriafurone, 8-O-methyl13-hydroxynorjavanicin, 8-O-methylanhydrofusarubinlactol, and 13-hydroxynorjavanicin), and a novel isoquinoline-type, pigment 2-(4-((3E,5E)-14-aminotetradeca-3,5-dienyloxy)butyl)-1,2,3,4tetrahydroisoquinolin-4-ol (ATDBTHIQN), from Fusarium fujikuroi (formerly known as Fusarium moniliforme) (Figure 2) $[25,63,65]$. Similarly, differently colored naphthoquinones [bostrycoidin, 9-O-methylfusarubin, 5-O-methyljavanicin, 8-O-methylbostrycoidin, 1,4-naphthalenedione-3,8dihydroxy-5,7-dimethoxy-2-(2-oxopropyl), 5-O-methylsolaniol, and 9-O-methylanhydrofusarubin], two anthraquinones compounds [2-acetyl-3,8-dihydroxy-6-methoxy anthraquinone and 2-(1-hydroxyethyl)3,8-dihydroxy-6-methoxy anthraquinone], and polyketide pigment (bikaverin) were reported from Fusarium oxysporum (Figure 2) $[25,47,64,67]$. Another species of Fusarium, Fusarium graminearum, has 
been found to produce a variety of pigments such as 5-deoxybostrycoidin anthrone, 6-O-dimethyl-5deoxybostrycoidin anthrone, purpurfusarin, 6-O-demethyl-5-deoxybostrycoidin, 5-deoxybostrycoidin, and aurofusarin (Figure 2) [25,64,66,121].<smiles></smiles>

$\mathbf{R} 1=\mathrm{CH}_{3} ; \mathbf{R} 2=\mathbf{H}$ 8-O-methylbostrycoidin<smiles>[R]C(C)=CC1=C(C)C(=O)c2c(O)cc(OC)c(O)c2C1=O</smiles>

$\mathbf{R} 1=\mathbf{C H}_{3} ; \mathbf{R} 2=\mathbf{O}$

8-O-methyljavanicin<smiles>[R]C(C)CC1=C(C)C(=O)c2c(O)cc(OC)c(O)c2C1=O</smiles>

$\mathbf{R} 1=\mathrm{CH}_{3} ; \mathbf{R 2}=\mathbf{O H}$

8-O-methylsolaniol<smiles>COc1cc(O)c2c(c1O)C(=O)C1=C(COC(C)(O)O1)C2=O</smiles>

$\mathbf{R 1}=\mathrm{CH}_{3} ; \mathbf{R 2}=\mathbf{H}$

8-O-methylfusarubin<smiles>[R]c1cc2c(cc1O)C(=O)c1cc(OC)cc(O)c1C2=O</smiles>

2-acetyl-3,8-dihydroxy-6methoxy anthroquinone<smiles>COc1cc(OC)c2c(c1)C1(CCCCC1)C(=O)c1cnc(C)cc1C2</smiles><smiles>Cc1cc2c(cn1)C(=O)c1c(O)cc(O)cc1C2</smiles>

6-O-demethyl-5deoxybostrycoidin anthrone

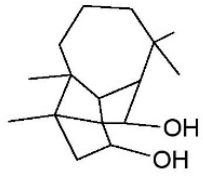

Culmorin<smiles>COC1C(=O)C(O)=C(O)c2c(O)c3c(c(O)c2C1=O)C(OC)C(O)C(O)C3O</smiles>

Benzoquinone

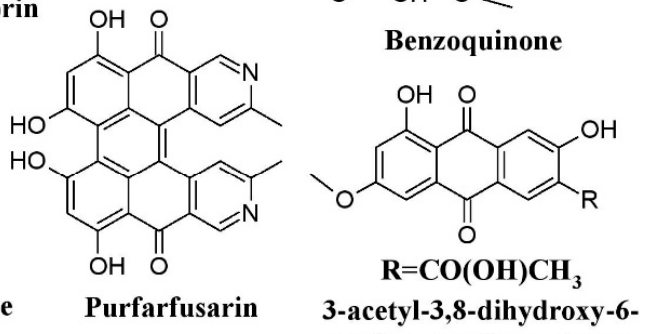
methoxy anthroquinone<smiles>NCCCCCCCOCCCCCCCN1Cc2ccccc2C(O)C1</smiles>

2-(4-((3E,5E)-14-aminotetradeca3,5-dienyloxy)butyl)-1,2,3,4tetrahydroisoquinolin-4-ol (ATDBTHIQN)<smiles>CC(C)=CC=CC(C)=CC=CC(C)=CC=CC(C)=CCC=C(C)CC=CC=C(C)C=CC=C(C)C=CC1=C(C)CCCC1(C)C</smiles>

(ATDBTHIQN)<smiles>COC1=CC(=O)C2C=C3OC(C)(O)OCC3C=C2C1=O</smiles>

Fusarubin<smiles>COc1cc(O)c2c(c1O)C(=O)C1COC(C)=CC1C2=O</smiles>

Anhydrofusarubin<smiles>CC1(O)Cc2c(O)c3c(c(O)c2CO1)C(=O)C(O)CC3=O</smiles>

$O$-demethyl fusarubin<smiles>COC1=CC(=O)c2c(O)c(C)c(CC(C)=O)c(O)c2C1=O</smiles>

Javanicin<smiles>COC1=CC(=O)C2C(=O)C=C(C(CO)C(C)=O)C(=O)C2C1=O</smiles>

Novarubin<smiles>COC1CC(=O)c2c(O)c(C)c3cc(C)oc3c2C1=O</smiles>

Anhydrojavanicin<smiles>CC(=O)Cc1c(C)c(O)c2c(c1O)C(=O)C(O)CC2=O</smiles>

$O$-demethyl javanicin<smiles></smiles>

Bikaverin<smiles>COc1cc(O)c2c(c1O)C(=O)C1C=C(C)N=CC1C2=O</smiles>

Bostrycoidin<smiles>CC1=CC2C(=O)c3c(O)c(O)cc(O)c3C(=O)C2C=N1</smiles>

O-demethyl bostrycoidin<smiles>C/C=C/C(=O)C=C(C)O/C=C/C1C(=O)C(=O)C(C2=C(OC)C3C=c4c(=O)cc(C)oc4=CC3C(=O)C2OC)C(=O)C1=O</smiles>

Aurofusarin<smiles>COc1cc(O)c2c(O)c3c(=O)cc(C)oc3cc2c1</smiles>

Rubrofusarin

Figure 2. Pigments from fungal genera of Nectriaceae (Fusarium, Fusicolla, and Albonectria), re-drawn from $[25,47,63,65,66,68]$. 
A red pigment aurofusarin has been found to be produced by many species of Fusarium such as Fusarium culmorum, Fusarium sporotrichioides, Fusarim. acuminatum, Fusarium avenaceum, Fusarium poae, Fusarium crookwellens, Fusarium pseudograminearum, Fusarium sambucinum, and Fusarium tricinctum. Bikaverin has been reported to be produced by Fusarium lycopersici, and Fusarium vasinfectum. Fusarium solani and Fusarium verticillioides (currently known as F. fujikuroi) have been described to produce both aurofusarin and bikaverin (Figure 2) [25]. Similarly, benzoquinone has been reported from Fusarium sp. JN158 (Figure 2) [68]. A study has shown that the synthesis of major Fusarium carotenoids (neurosporaxanthin and $\beta$-carotene) is induced by light via transcriptional induction of the structural genes carRA, carB, carT, and carD [43]. Similarly, other members of the fungal family Nectriaceae, such as Albonectria rigidiuscula and Fusicolla aquaeductuum (formerly known as Fusarium decemcellulare and Fusarium aquaeductuum respectively) were reported for their pigment production potential (Figure 2) [43,64]. Recently, the biosynthetic pathway of chrysogine mediated by two-module non-ribosomal peptide synthetase (NRPS) gene cluster was discovered in Fusarium graminearum in which enhanced chrysogine production was observed upon overexpression of NRPS14 [122].

Many investigations report Penicillium as potent producers of pigment [25,61,96-98], such as arpink $\operatorname{red}^{\mathrm{TM}}$ (first commercial red colorant), talaroconvolutins A-D, sclerotiorin, xanthoepocin, atrovenetin, and dihydrotrichodimerol discovered from Penicillum oxalicum var. armeniaca, Penicillum convolutum (formerly known as Talaromyces convolutes), Penicillum mallochii, Penicillum simplicissimum, Penicillum melinii, and Penicillum flavigenum, respectively (Figure 3a) [41,91,93,94,123]. An uncharacterized red pigment has been reported from Penicillium miczynskii [71]. Besides, many other Monascus-like pigments such as PP-V [(10Z)-12-carboxylmonascorubramine] and PP-R [(10Z)-7-(2-hydroxyethyl)monascorubramine] have been reported from Penicillium (Figure 4) [95]. A biosynthetic pathway for the yellow pigment chrysogine from Penicillium chrysogenum has been proposed recently [92].

Talaromyces spp. have been reported as a source of pigments by many researchers. The pigment production ability of Talaromyces purpureogenus (formerly known as Penicillium purpureogenum) was evaluated by many researchers [102,104,105]. Studies report the production of a herqueinone-like pigment from Talaromyces marneffei (formerly known as Penicillium marneffei), Monascus-like azaphilone pigments ( $N$-glutarylmonascorubramine and $N$-glutarylrubropunctamine) from Talaromyces purpureogenus (formerly known as Penicillium purpureogenum), industrially important red pigments (mitorubrin, monascorubrin, PP-R, glauconic acid, purpuride, and ZG-1494 $\alpha$ ) from Talaromyces atroroseus, trihydroxyanthraquinones (emodin, erythroglaucin, and catenarin) from Talaromyces stipitatus, and a xanthone dimer (talaroxanthone) from Talaromyces sp. (Figure 3b) [100,101,103,107,109]. An uncharacterized red pigment was discovered from Talaromyces siamensis under submerged fermentation [71]. Moreover, other species of Talaromyces, Talaromyces aculeatus, Talaromyces atroroseus, Talaromyces albobiverticillius, Talaromyces cnidii, Talaromyces coalescens, Talaromyces pinophilus, Talaromyces purpurogenus, Talaromyces funiculosus, Talaromyces amestolkiae, Talaromyces ruber, Talaromyces stollii, and Talaromyces verruculosus have been reported to have the ability to produce Monascus-like azaphilone pigments (Figure 4) [25,106].

Several members of the genus Aspergillus, such as Aspergillus niger, have been known to synthesize a wide variety of pigments, such as aspergillin, asperenone, azaphilones (azanigerones A-F), and melanin (Figure 5a) [25,110,114,115]. Aspergillus nidulans was reported to produce ascoquinone A, norsolorinic acid, and melanin [25,112,113], whereas Aspergillus fumigatus was reported to produce melanin and melanin-like pigments [25,111]. In addition, a variety of other pigments such as asperenone, anishidiol, neoaspergillic acid, sterigmatocystin, and an uncharacterized yellow pigment have been discovered from Aspergillus nishimurae, Aspergillus awamori, Aspergillus sclerotiorum, Aspergillus versicolor, and Aspergillus terreus, respectively [25,91,110,116,118]. Many other species of Aspergillus such as Aspergillus glaucus, Aspergillus cristatus, and Aspergillus repens have been reported to produce a variety of hydroxyanthraquinone pigments, emodin, physcion, questin, erythroglaucin, catenarin, and rubrocristin; while Aspergillus melleus, Aspergillus ochraceus, Aspergillus sulphureus, and Aspergillus 
westerdijkiae have been described to be major producers of polyketide-based pigments (rubrosulfin, viomellein, viopurpurin, and xanthomegnin) (Figure 5a) [25]. In addition to this, other pigments such as ferriaspergillin, ferrineoaspergillin, and an uncharacterized yellow pigment have also been reported from the genus Aspergillus (Figure 5a) [119,120].

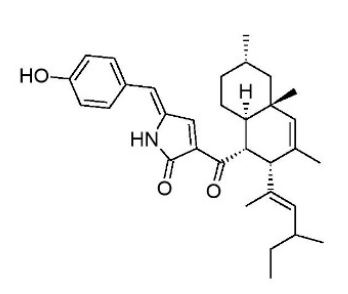

Talaroconvolutin A

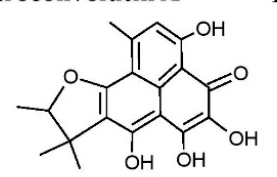

Xanthoepoein

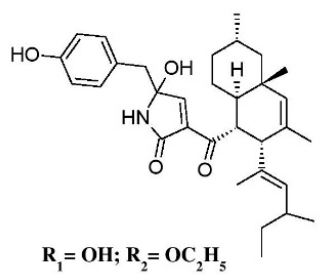

Talaroconvolutin B

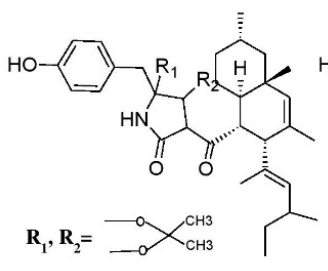

Talaroconvolutin C

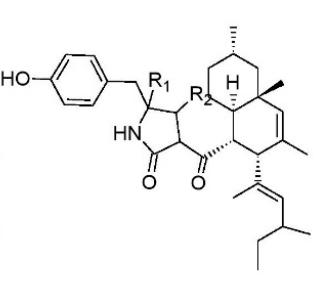

Talaroconvolutin D

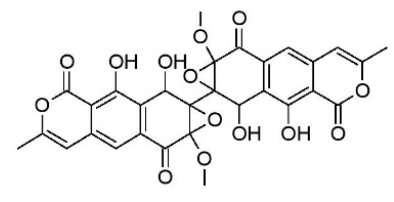

Atrovenetin

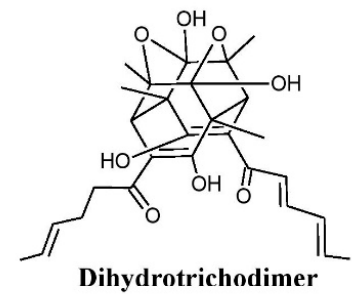

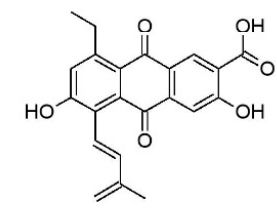

Arpink red

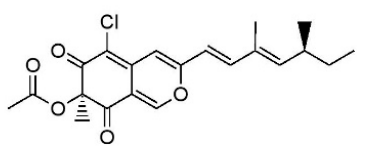

Sclerotiorin

(a)<smiles>C/C=C/C1=CC2=CC(=O)C(C)(OC(=O)c3c(C)cc(O)cc3O)C(=O)C2=CO1</smiles>

Mitorubrin

Monascorubin

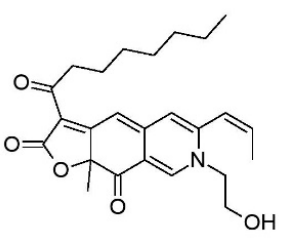

PP-R<smiles>CCC1C=C2C(=O)OC(=O)[C@@]2(C)CC2=C1[C@H](O)C(O)[C@H](CC)C(=O)OC2=O</smiles>

Emodin

Glauconic acid

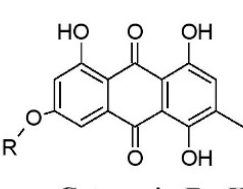

Catenarin $\mathbf{R}=\mathbf{H}$ Erythroglaucin $\mathrm{R}=\mathrm{CH}_{3}$<smiles>O=C(O)c1cc(O)c2c(c1)C(=O)c1cc(O)cc(O)c1C2=O</smiles>

Emodic acid

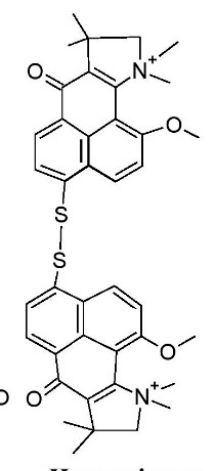

Herqueinonelike pigment<smiles>CCC(C)/C=C(\C)[C@H]1C(C)=C[C@]2(C)C[C@H](C)CC[C@H]2[C@H]1C(=O)C1=CC(O)(Cc2ccc(O)cc2)NC1=O</smiles><smiles>O=C1c2cc(CO)cc(O)c2C(=O)c2cc(O)cc(O)c21</smiles>

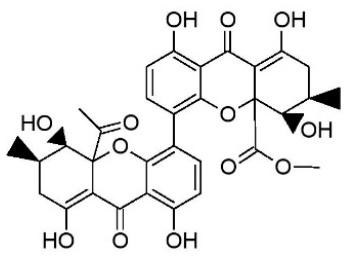

$\omega$-hydroxyemodin

Talaroxanthone

(b)

Figure 3. Pigments from the genera Penicillium and Talaromyces. (a) Different pigments produced by Penicillium species, re-drawn from $[41,91,93,94,123]$. (b) Various pigments produced by Talaromyces species, re-drawn from $[100,101,107,109]$. 


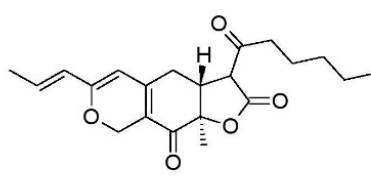

Monascin

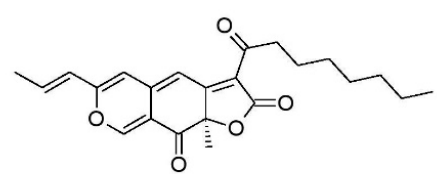

Monascorubrin

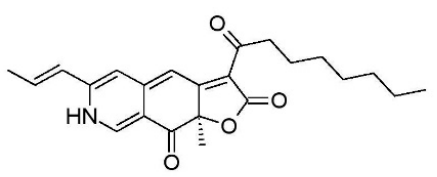

Monascorubramine<smiles>C/C=C/C1=CC2=CC3=C(C(=O)CCCCC)C(=O)OC3(C)C(=O)C2=CO1</smiles>

Rubropunctatin

Ankaflavin<smiles>C/C=C/C1=CC2=CC3=C(C(=O)CCCCC)C(=O)O[C@@H]3C(=O)C2=CN1C(CCC(=O)O)CCC(=O)O</smiles>

$N$-glutarylrubropunctamine

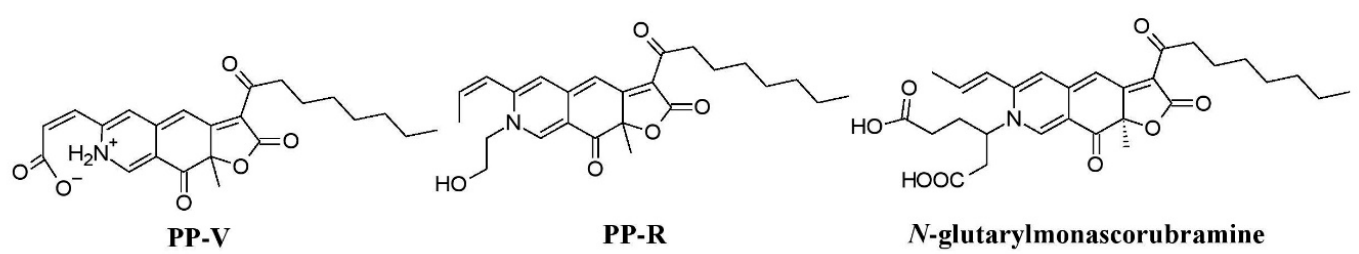

Figure 4. Monascus-like azaphilone pigments of Penicillium and Talaromyces species, re-drawn from $[25,95,106]$.

Certain teleomorphic species of Aspergillus have been described as producers of a variety of pigments. Some of the well-known azaphilone pigments such as falconensins A-H, zeorin, falconensones A1 and B2 have been reported from Emericella falconensis and Emericella fruticulosa (currently known as Aspergillus falconensis and Aspergillus fruticulosus, respectively), epurpurins A-C from Emericella purpurea (currently known as Aspergillus purpureus), and the pigment sterigmatocystin from Emericella rugulosus, Emericella parvathecia, and Emericella nidulans (currently known as Aspergillus rugulosus, Aspergillus parvathecia, and Aspergillus nidulans) (Figure 5c). Similarly, other Aspergillus spp. such as Aspergillus amstelodami, Aspergillus chevalieri, Aspergillus glaucus, Aspergillus umbrosus, Aspergillus spiculosus, Aspergillus glaber, Aspergillus echinulatum, Aspergillus tonophilus, Aspergillus intermedius, Aspergillus leucocarpus, Aspergillus ruber, and Aspergillus cristatus (which were formerly known as Eurotium amstelodami, Eurotium chevalieri, Eurotium herbariorum, Eurotium umbrosum, Eurotium spiculosum, Eurotium spiculosum, Eurotium echinulatum, Eurotium tonophilum, Eurotium intermedium, Eurotium leucocarpum, Eurotium rubrum, and Eurotium cristatum, respectively) have also been reported to produce pigments such as physcion, erythroglaucin, flavoglaucin, auroglaucin, catenarin, rubrocristin, and emodin (Figure 5b) [25].

Members of different genera of the fungal family Pleosporaceae (Alternaria, Curvularia, Pyrenophora, etc.) have immense potential for pigment production. Species of Alternaria such as Alternaria alternata, Alternaria solani, Alternaria porri, and Alternaria tomatophila have been reported to produce a variety of pigments such as dactylariol, alterperylenol, dihydroalterperylenol, alternariol, alternariol-5-methyl ether, altenuene, alternarienoic acid, tenuazoic acid, stemphyperylenol, and altersolanol A (Figure 6) [25,76-78]. Also, other members of the Pleosporaceae, Curvularia and Pyrenophora, have been known to produce different types of pigments, e.g., Curvularia lunata produces hydroxyanthraquinone pigments such as chrysophanol, cynodontin, helminthosporin, erythroglaucin, and catenarin, whereas different species of Pyrenophora such as Pyrenophora teres, Pyrenophora graminea, Pyrenophora tritici-repentis, Pyrenophora grahamii, Pyrenophora dictyoides, and Pyrenophora chaetomioides (which were previously known as Drechslera teres, Drechslera graminea, Drechslera tritici-repentis, Drechslera phlei, Drechslera dictyoides, Drechslera avenae, respectively) have also been reported to produce hydroxyanthraquinone pigments such as cynodontin, erythroglaucin, catenarin, helminthosporin, and tritisporin (Figure 6) [25,61]. Trichoderma, a well-known bio-control agent, has been known to produce a variety of pigments $[25,124]$. Several hydroxyanthraquinones such as pachybasin, chrysophanol, emodin, T22 azaphilone, 1-hydroxy-3-methyl-anthraquinone, 2,4,5,7-tetrahydroxyanthraquinone, 
1,3,6,8-tetrahydroxyanthraquinone, and 1,8-dihydroxy-3-methyl-anthraquinone, have been reported from different species of Trichoderma (Trichoderma harzianum, Trichoderma polysporum, Trichoderma viride, and Trichoderma aureoviride) (Figure 7a) [25], whereas Trichoderma afrharzianum, Trichoderma pyramidale, and Trichoderma sp. 1 are reported to produce uncharacterized yellow pigments in submerged fermentation [71]. Studies have also revealed that certain species of Neurospora, such as Neurospora crassa, Neurospora sitophila, and Neurospora intermedia produce a variety of carotenoids such as phytoene, $\beta$-carotene, $\gamma$-carotene, lycopene, neurosporene, and neurosporaxanthin (Figure $7 b)[25,26,90]$.

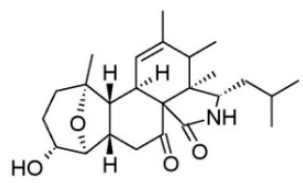

Aspergillin PZ<smiles>CC1=Cc2c(O)c(C)c(O)c(C=O)c2CO1</smiles>

Anishidiol

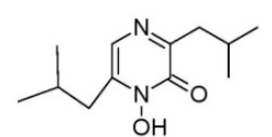

Ferrineoaspergillin

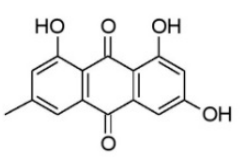

Emodin

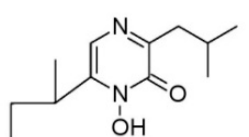

Ferriaspergillin<smiles>COc1cc(O)c2c(c1)C(=O)c1cc(C)cc(O)c1C2=O</smiles>

Physcion<smiles>O=c1oc2cccc(O)c2c(=O)c2c(O)cccc12</smiles>

Hydroxylated anthroquinone

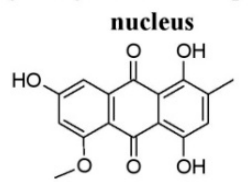

Rubrocristin<smiles>CCC(C)CC(C)C(=O)OC1(C)C(=O)C=C2C=C(CC(C)O)OC=C2C1=O</smiles>

Azanigerone B

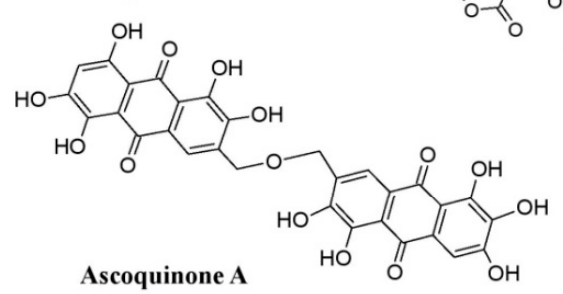

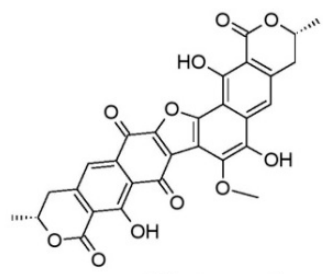

Viopurpurine<smiles>CCC(C)CC(C)C(=O)OC1(C)C(=O)C=C2C=C(C(O)C(C)O)OC=C2C1=O</smiles>

Azanigerone C<smiles>CC(C)Cc1ncc(CC(C)C)n(O)c1=O</smiles>

Neoaspergillic acid

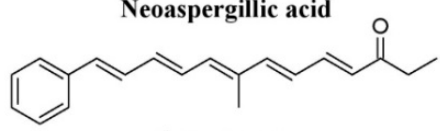

Asperenone

(a)

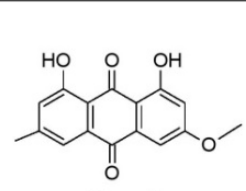

Physcion

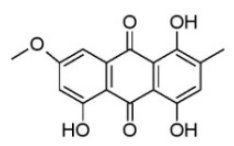

Erythroglaucin<smiles>[R]c1c(CC)cc(O)c2c1C(=O)c1cc(O)cc(O)c1C2=O</smiles>

Emodin $\mathbf{R}=\mathbf{H}$

Catenarin $\mathbf{R}=\mathbf{O H}$

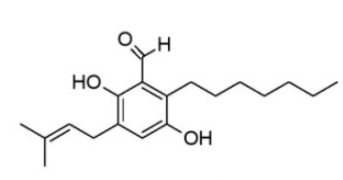

Flavoglaucin

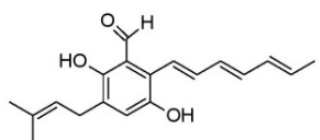

Auroglaucin

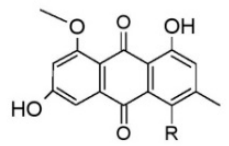

Questin R= H

Rubrocristin $\mathbf{R}=\mathbf{O H}$

(b)

Falconensin A<smiles>[R][R]([H])=C[R](=C)C</smiles><smiles>[R]Oc1ccc(/C=C(C)/C(C)=C/c2ccc(O[R])c([R])c2)cc1[R]</smiles>

Epurpurin A $\mathbf{R}_{1}=\mathrm{H}, \mathrm{R}_{2}=\mathrm{R}_{3}=\mathrm{CH}_{2} \mathrm{CH}_{2} \mathrm{CHC}\left(\mathrm{CH}_{3}\right)_{2}$ Epurpurin B $R_{1}=R_{2}=H, R_{3}=\mathrm{CH}_{2} \mathrm{CH}_{2} \mathrm{CHC}\left(\mathrm{CH}_{3}\right)_{2}$ Epurpurin $C \quad R_{1}=R_{2}=R_{3}=H$

(c)

Figure 5. Pigments from the genus Aspergillus and its teleomorphic genera. (a) Structures of pigments produced by Aspergillus species. (b) Pigments produced by species of Eurotium (teleomorph of Aspergillus). (c) Pigments produced by species of Emericella (teleomorph of Aspergillus), re-drawn from [25]. 


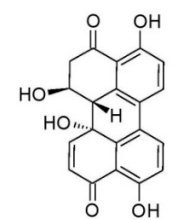

Alterperylenol<smiles>Cc1cc(O)cc2oc(=O)c3c(O)cc(O)cc3c12</smiles>

Alternariol

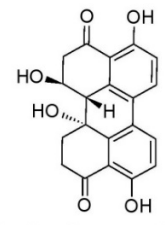

Dihydroalterperylenol

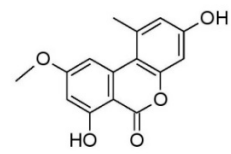

Alternariol 5 methyl ether

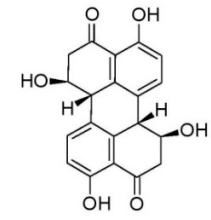

Stemphyperylenol

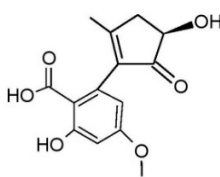

Alternarienoic acid

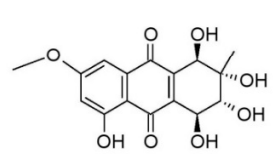

Altersolanol A

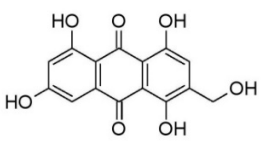

Tritisporin<smiles>CCC(=O)CC1C(=O)c2c(OC)cc(OC)cc2C(=O)C1C</smiles>

Astropaquinone A

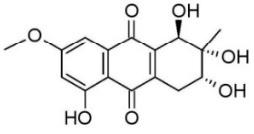

Dactylariol

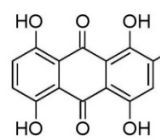

Cynodontin

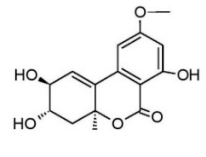<smiles>CCC(C)NC(=O)/C(C=O)=C/CO</smiles>

Tenuazoic acid

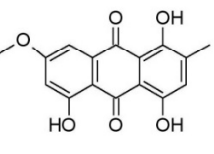

Erythroglaucin

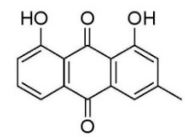

Chrysophanol

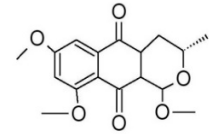

Astropaquinone B

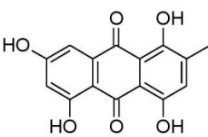

Catenarin

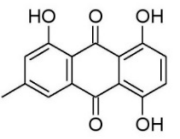

Helminthosporin

Figure 6. Pigments produced by members of the fungal family Pleosporaceae (species of Alternaria, Curvularia, Astrosphaeriella, and Pyrenophora), re-drawn from [25,76-78].

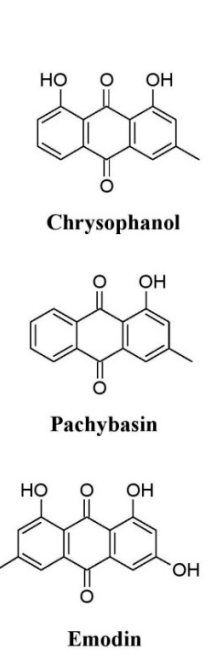

(a)
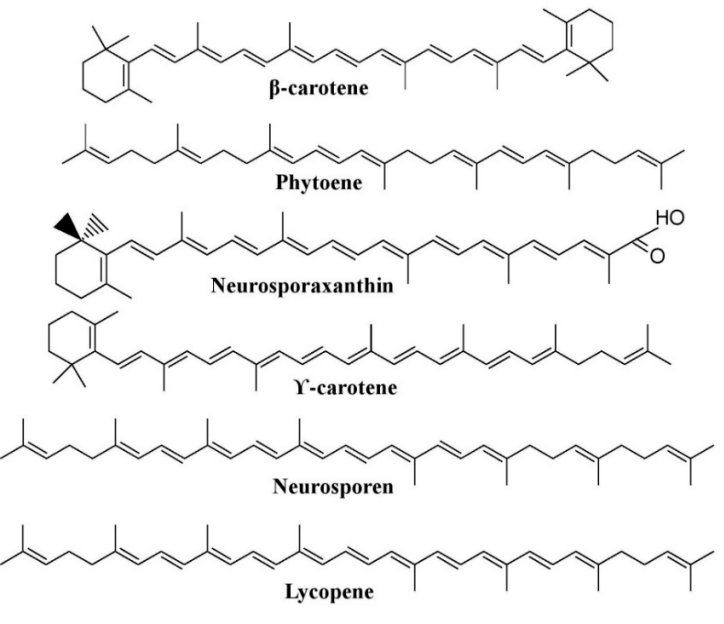

(b)

Figure 7. Pigments from other fungi. (a) Pigments from Trichoderma species, based on [25]. (b) Pigments from Neurospora species, re-drawn from $[25,90]$.

Many genera of the Xylariaceae family, such as Daldinia, Hypoxylon, Jackrogersella, etc., have a great capability to synthesize pigments of very diverse colors and hues [25]. A variety of interesting pigments such as BNT (1,1'-Binaphthalene-4,4' $-5,5^{\prime}$-tetrol), daldinol, daldinal A-C, and daldinin A-C have been reported from different species of Daldinia, such as Daldinia bambusicola, Daldinia caldariorum, Daldinia concentrica, Daldinia eschscholzii, Daldinia childiae, Daldinia clavata, Daldinia fissa, Daldinia grandis, Daldinia 
lloydi, Daldinia loculata, Daldinia petriniae, Daldinia singularis (Figure 8a). Similarly, several cohaerin variants (cohaerin A-K), multiformin A, and sassafrins D have been obtained from Jackrogersella cohaerens (formerly known as Annulohypoxylon cohaerens) (Figure 8a). Besides this, several species of Hypoxylon were declared to produce diverse pigments e.g., Hypoxylon fragiforme (hypoxyxylerone, cytochalasin $\mathrm{H}$, fragiformins $\mathrm{A}-\mathrm{B}$, and mitorubrin), Hypoxylon howeanum (mitorubrin and azaphilones), Hypoxylon lechatii (vermelhotin and hypoxyvermelhotins A-C), Hypoxylon fuscum (daldinin A-C), Hypoxylon fulvo-sulphureum (mitorubrinol derivatives), Hypoxylon sclerophaeum (hypoxylone), Hypoxylon rickii (rickenyl B and D), Hypoxylon lenormandii and Hypoxylon jaklitschii (lenormandins A-G), Hypoxylon rubiginosum (mitorubrin, rubiginosin, and hypomiltin) (Figure 8a). Members of the Chaetomiaceae family also exhibit potential of pigment production. Chaetomium cupreum has been mentioned to produce red azaphilone pigments, oosporein, rotiorinols $\mathrm{A}-\mathrm{C}$, rubrorotiorin, whereas Chaetomium globosum produces yellow azaphilone pigments (chaetoviridins A-D), chaetoglobin A-B, chaetomugilins A-F, and cochliodinol (Figure $8 \mathrm{~b}$ ). Production of parietin (hydroxyanthraquinone pigment) has also been revealed from the Achaetomium sp. (Figure 8b) [25].
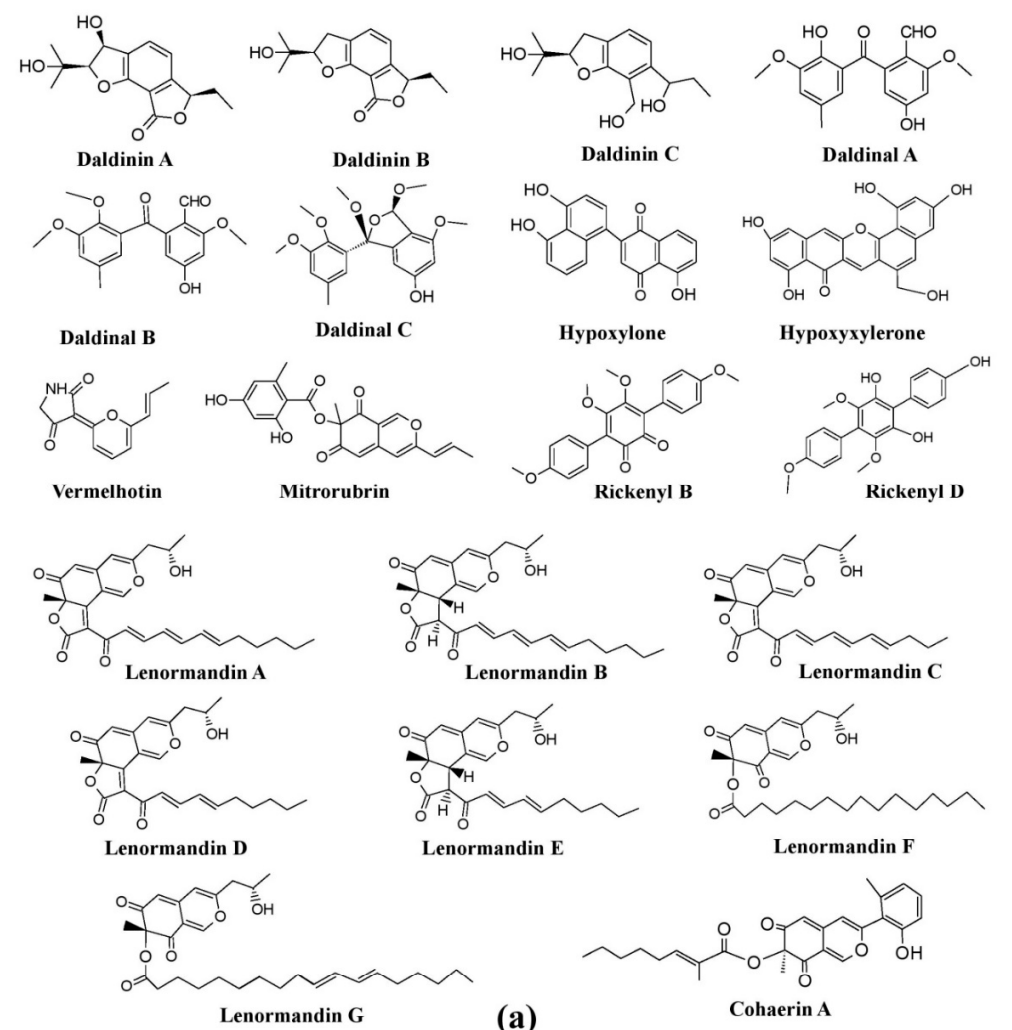

(a)

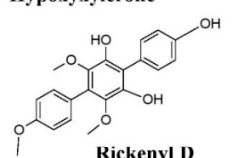

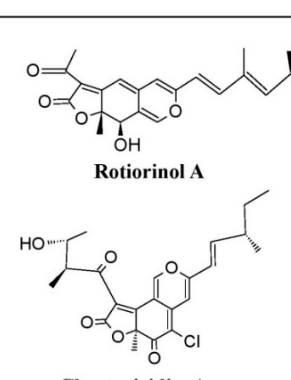

Chaetoviridin A

Cohaerin A
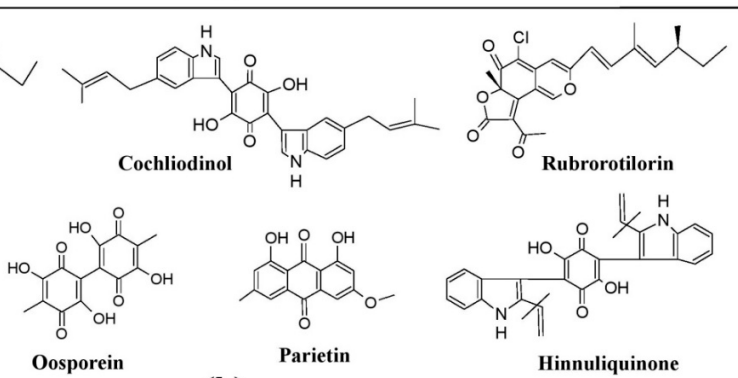

(b)

Figure 8. Pigments from the fungi of Xylariaceae and Chaetomiaceae families. (a) Pigments from members of the Xylariaceae family (species of Daldinia, Hypoxylon, and Jackrogersella), re-drawn from [25]. (b) Pigments from members of the Chaetomiaceae family (species of Chaetomium and Achaetomium) and Hypoxylaceae, re-drawn from $[25,84]$. 
Also, the genera belonging to the family Cordycipitaceae such as Torrubiella, Cordyceps, Beauveria, Hyperdermium, and Lecanicillium have been revealed to be promising producers of bioactive pigments, e.g., tenellin and bassianin are reported from Beauveria bassiana and Beauveria brongniartii (formerly known as Beauveria tenella), pyridovericin and pyridomacrolidin from Beauveria bassiana, torrubiellones A-D from the genus Torubiella, oosporein from Lecanicillium aphanocladii, whereas anthraquinone-related compounds are reported from Cordyceps farinosa (formerly known as Isaria farinosa) (Figure 9a) [41,73-75,125]. Similarly, the pigments erythrostominone, 4-O-methyl erythrostominone, deoxyerythrostominone, deoxyerythrostominol, epierythrostominol, and 3,5,8-TMON (3,5,8-trihydroxy-6-methoxy-2-(5-oxohexa-1,3-dienyl)-1,4-naphthoquinone) have been reported from Ophiocordyceps unilateralis (formerly known as Cordyceps unilateralis), and skyrin from Hyperdermium bertonii (Figure 9a) [25].
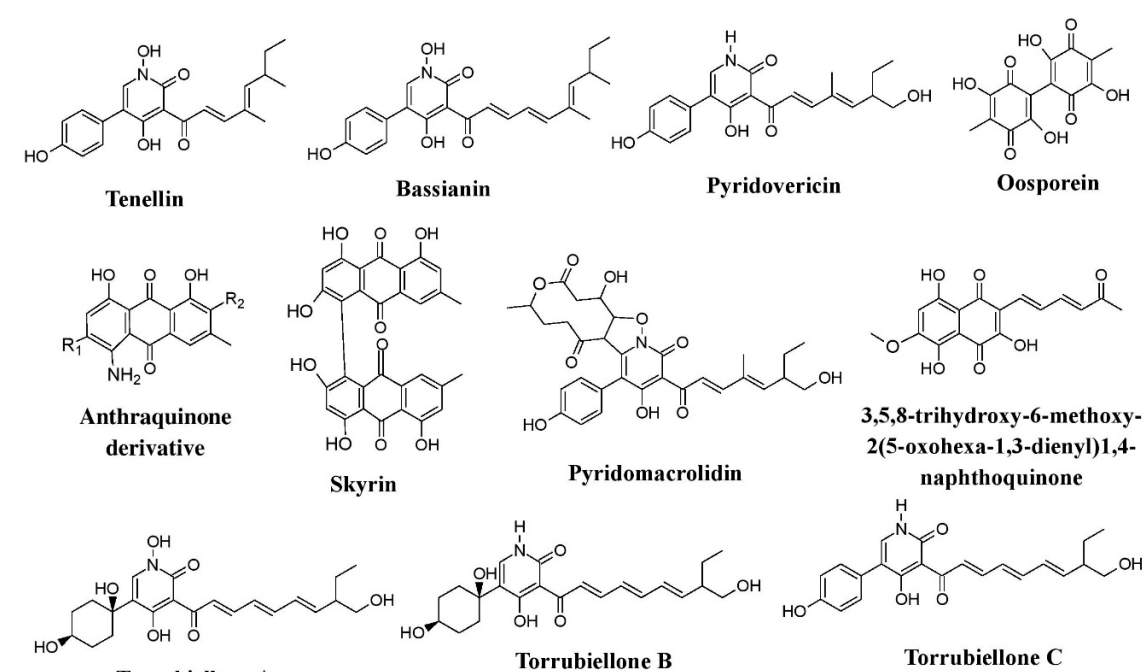<smiles>CC=Cc1ccc(O)cc1</smiles>

H

Torrubiellone A

Torrubiellone B

Torrubiellone C
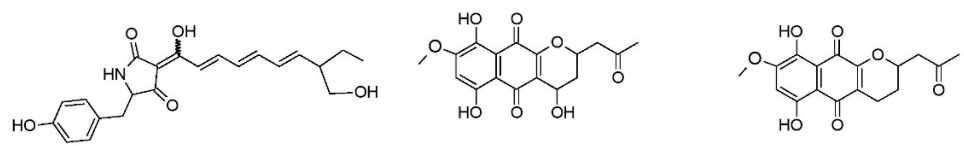

Torrubiellone D

Erythrostominone

Deoxyerythrostominone
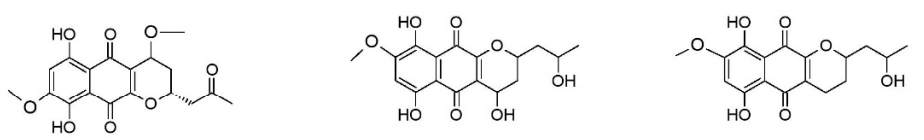

4-O-methyl erythrostominone

Epierythrostomino

Deoxyerythrostominonol

(a)

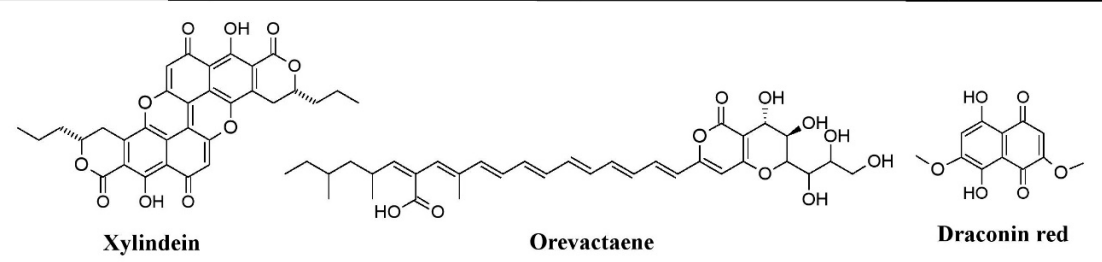

(b)

Figure 9. Pigments from the fungi of the Cordycipitaceae family and some other group. (a) Pigments from members of the families Cordycipitaceae (species of Beauveria, Torrubiella, Cordyceps, Hyperdermium, and Lecanicillium) and Ophiocordycipitaceae (Ophiocordyceps sp.), re-drawn from [25,41,73-75,125]. (b) Pigments known from other groups of fungi (species of Chlorociboria, Scytalidium, and Epicoccum), re-drawn from $[37,41]$. 
Apart from this, studies have reported the production of the pigment xylindein from Chlorociboria aeruginosa and Chlorociboria aeruginascens, draconin red from Scytalidium cuboideum, and a yellow pigment from Scytalidiium ganodermophthorum and Scytalidium lignicola. Other pigments, such as orevactaene produced from Epicoccum nigrum, emodin, $\omega$-hydroxyemodin, and emodic acid from Hamigera avellanea (formerly known as Talaromyces avellaneus) are also known (Figure 3b, Figure 9b) [33,36,37,39,41,109]. Recently, fungi such as Sanghuangporus baumii and Clonostachys intermedia have been found to produce a yellow pigment under submerged fermentation [71]. Production of melanin was reported from different groups of fungi such as Phyllosticta capitalensis, Xylaria polymorpha, Trametes versicolor, Inonotus hispidus, Oxyporus populinus, Fomes fomentarius, Exophiala dermatitidis, Tuber melanosporum, Sporothrix schenckii, and Cryptococcus neoformans [29,34,35,44,80,81,83]. Similarly, a study has shown the possible industrial application of the red pigment produced by Paecilomyces sinclairii [126]. Besides filamentous fungi, certain genera of yeasts (Rhodotorula, Sporidiobolus, Sporobolomyces and Xanthophyllomyces) have also been known as pigment producers. Different species of Rhodotorula (Rhodotorula glutinis, Rhodotorula mucilaginosa (syn. Rhodotorula rubra), Rhodotorula babjevae, Rhodotorula toruloides Rhodotorula graminis), Sporidiobolus (Sporidiobolus pararoseus, Sporidiobolus johnsonii), and Sporobolomyces (Sporobolomyces uberrimus, Sporobolomyces salmonicolor) have been reported to be prolific producers of torulin and torularhodin [127]. Researchers have discovered pigments such as $\beta$-carotene, torulene, and torularhodin from Rhodotorula glutini and multi-hydroxy carotenoids $\left(4,4^{\prime}\right.$-dihydroxy-nostoxanthin and 4-hydroxy-nostoxanthin) from Xanthophyllomyces dendrorhous (Figure 10) [13,128].
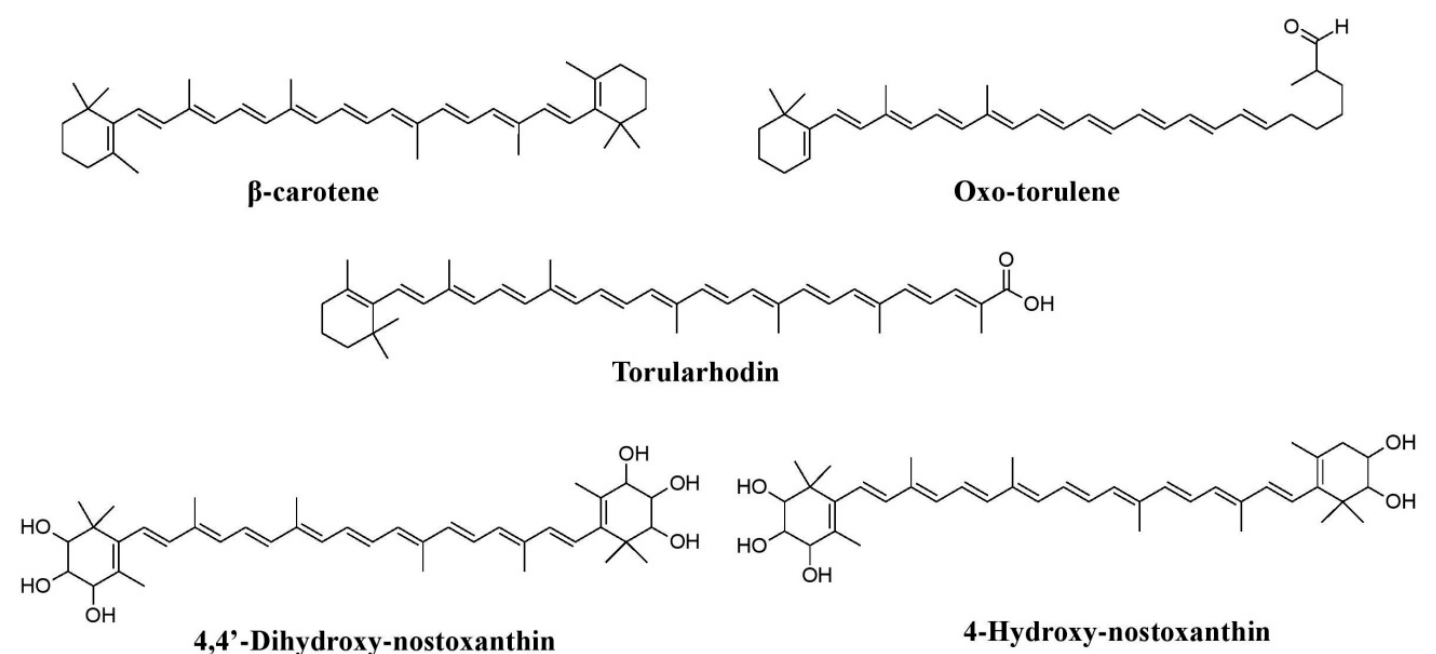

Figure 10. Pigments reported from yeasts such as Rhodotorula glutini and Xanthophyllomyces dendrorhous, re-drawn from $[13,128]$.

In addition to terrestrial fungi, marine fungi are also very good producers of a variety of unique pigments having promising therapeutic and industrial applications [129,130]. Studies on marine fungi by many researchers have reported a wide range of pigments and hues, e.g., a variety of anthraquinone pigments [asperflavin, 2-O-methyleurotinone, questin, eurorubrin, 2-O-methyl-9-dehydroxyeurotinone, 2-O-methyl- 4-O-( $\alpha$-D-ribofuranosyl)-9-dehydroxyeurotinone, and 6, 3-O-( $\alpha$-D-ribofuranosyl)-questin] from the mangrove endophytic fungus $A$. ruber (formerly known as Eurotium rubrum), fusarnaphthoquinones B and fusarnaphthoquinones $C$ from the sea fan-derived fungi Fusarium species, and bianthraquinone derivatives (alterporriol K, alterporriol L, and alterporriol M) from mangrove endophytic Alternaria sp. (Figure 11) [69,79,117]. Researchers have also investigated the red pigment production from mangrove fungus Penicillium sp. and a yellow pigment production from the marine sponge-associated fungus Trichoderma parareesei $[70,99]$. 


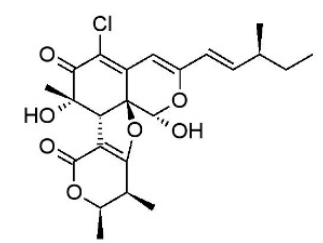

Chaephilone C

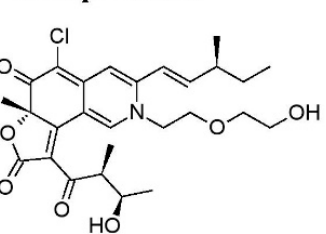

Chaetoviridin B

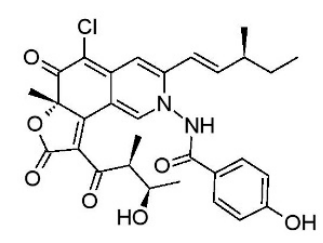

Chaetoviridide A

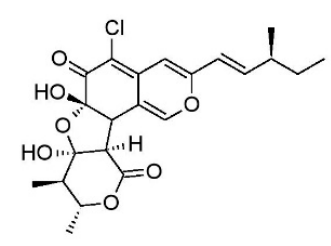

Chaetomugilin D

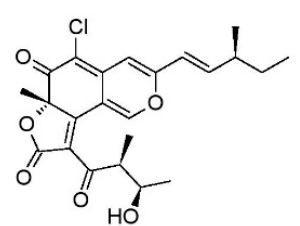

Chaetoviridin A<smiles>CCC(=O)C1=C(Cl)C2=CC(=C(C(=O)CC)C1(C)O)N(CCOCCO)C(C=CC(C)CC)=C2</smiles>

Chaetoviridin C

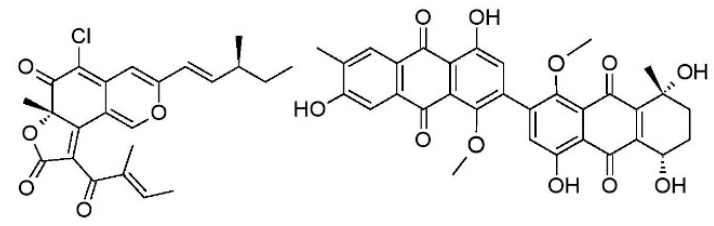

Chaetoviridin E

Alterporriol K

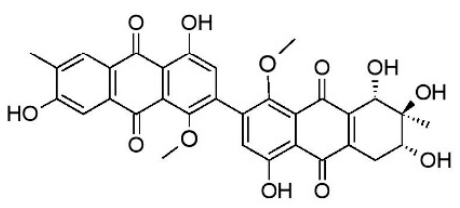

Alterporriol L

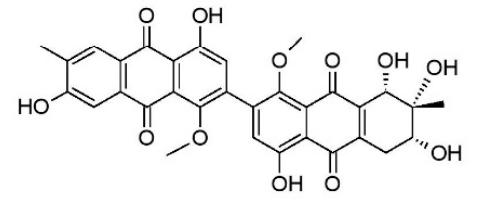

Alterporriol M<smiles>COC1CC(=O)c2c(O)c(C)c3cc(C)oc3c2[C@H]1O</smiles>

Fusarnaphthoquinone B

Fusarnaphthoquinone C<smiles>CC(C)[C@H](C)/C=C/[C@H](C)[C@H]1CCC2C3CC=C4C[C@@H](O)CC[C@]4(C)C3CCC21C</smiles><smiles>[R]Oc1cc(OC)c2c(c1)C(=O)c1c(O)cc(C)cc1C2=O</smiles>

Anthraquinone glycoside $\mathrm{R}=\alpha$-D-ribofuranosyl<smiles>COc1cc(O)cc2cc3c(c(O)c12)C(=O)CC(C)(O)C3</smiles><smiles>C/C=C/C1=CC2=C(CN1CC(=O)O)C(=O)[C@@]1(C)OC(=O)C(C(=O)CCCCC)=C1C2</smiles>

Asperflavin

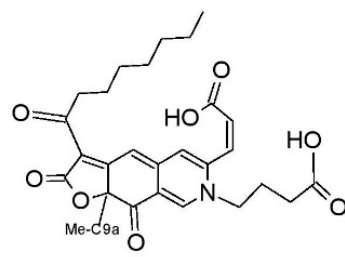

N-GABA- PP-V (6-I(Z)-2Carboxyvinyl]- $N$-GABAPP-V)

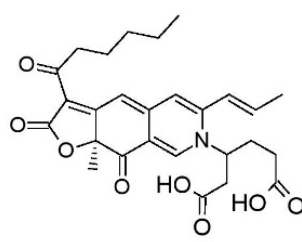

Ergosterol

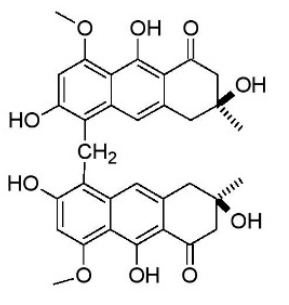

Eurorubrin

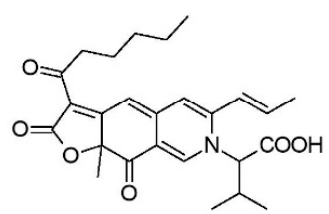

$N$-threoninerubropunctamine

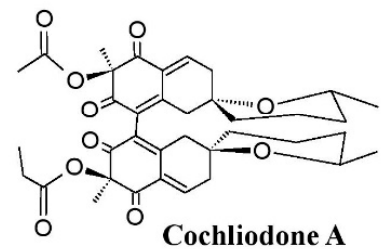

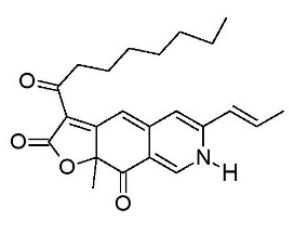$$
\begin{aligned}
& \text { 4,2-O-methyl4- } O \text {-( } \alpha \text {-D-ribofuranosyl)-! } \\
& \text { dehydroxyeurotinone } \\
& \left(\mathrm{R}_{1}=\alpha \text {-D-ribofuranosyl, } \mathrm{R}_{2}=\mathrm{H}\right)
\end{aligned}
$$

( $\mathrm{R}_{1}=\alpha$-D-ribofuranosyl, $\mathrm{R}_{2}=\mathrm{H}$ )

Seco-anthraquinone derivatives

3,2-O-methyl-9-dehydroxyeurotinone $\left(\mathrm{R}_{1}=\mathrm{R}_{2}=\mathrm{H}\right)$

2-O-methyleurotinone $\left(\mathrm{R}_{1}=\mathrm{R}_{2}=\mathrm{OH}\right)$

Figure 11. Pigments produced by marine fungal isolates, re-drawn from $[69,72,79,82,117]$.

Also, many studies have revealed the production of polyketide pigments ( $N$-threonine rubropunctamine) and chlorinated azaphilone pigments (chaephilone- $\mathrm{C}$, chaetoviridides- $\mathrm{A}$, chaetoviridides-B, chaetoviridides-C) from marine fungal isolates of Talaromyces spp. and Chaetomium sp., respectively (Figure 11) [72,82]. A recent study has reported a novel pigment, N-GABA-PP-V (6-[(Z)-2-Carboxyvinyl]-N-GABA-PP-V), along with $N$-threonine-monascorubramine, $N$-glutarylrubropunctamine, and $\mathrm{PP}-\mathrm{O}$ from the marine-derived fungus Talaromyces albobiverticillius (Figure 11) [131]. Many antarctic fungi have also been discovered to produces pigments of different 
chemical classes and characteristics. A number of yeast and filamentous fungi isolated from the different samples collected from Antarctic regions have been reported to produce a variety of pigments with different colors [86].

\section{Optimization for Enhancement of Pigment Production}

Most of the investigators have focused their study on the enhancement of pigment production from different fungal strains such as Monascus, Penicillium, Talaromyces, Fusarium, etc., by optimizing various fermentation parameters such as media, media composition, $\mathrm{pH}$, temperature, light intensity, orbital speed, etc. [26,132-135]. Some studies have reported about the assessment of the pigment production potential of different fungi on natural substrates (rice, corn, wheat, cassava, whole sorghum grain, dehulled sorghum grain, and sorghum bran) and on different agro-industrial residues (feather meal, fish meal, cheese whey, grape waste, soybean protein, soybean meal, chicken feather and rice husk, orange processing waste) [134,136-138]. Enhancement in xylindein production was reported in Chlorociboria aeruginascens upon addition of test woods (Acer saccharum, Populus tremuloides, spalted P. tremuloides, and Ailanthus altissima) in agar-based media [33].

Some studies have also evaluated the effect of different sugar sources such as glucose, fructose, lactose, sucrose, and maltose on pigment production by the species of Monascus. Results of these studies have shown that maximum pigment production was acheived in media with fructose as a carbon source for M. purpureus, and lactose as a carbon source for M. ruber [132,139]. Studies have also discovered that the addition of different nitrogen sources such as ammonium, peptone, sodium nitrate, glutamic acid, monosodium glutamate, 6-furturylaminopurine, and tryptophan could enhance the yield of pigment, alter the hue of the fermentation liquid, and also improve light stability of the pigments of Monascus species [132,140-143]. $\mathrm{NaCl}$ has been proved to be a very good enhancer that stimulates pigment production and inhibits citrinin production in $M$. purpureus without affecting the growth of the fungus [144]. A study on the effect of nutrients on pigment production of C. aeruginascens shows that high biomass but no pigment production was observed in media with high nutrient concentration, whereas low biomass and high pigmentation was observed in media with low nitrogen concentration [145]. Investigators have also found variations in the yield, color characteristics (hue and chroma values), and structure of the pigments of Monascus species with respect to the type of amino acids in the media [146,147]. Beside this, the $\mathrm{pH}$ of the media also plays an important role in pigment production. In the case of Monascus species (M. purpureus, M. major, and M. rubiginosus), $\mathrm{pH}$ optimization studies have shown that a low $\mathrm{pH}$ of the media increases pigment production $[140,146,148]$. Another study has revealed that the $\mathrm{pH}$ of the substrate plays an important role in melanin production by X. polymorpha, T. versicolor, Cerioporus squamosus (formerly known as Polyporus squamosus), Lentinus brumalis (formerly known as Polyporus brumalis), F. fomentarius and I. hispidus. The maximum pigment production was observed in the $\mathrm{pH}$ range from 4.5 to 5.5 [35]. Similar studies in other fungi such as Penicillium purpurogenum, P. aculeatum, A. niger, Altemaria sp., Fusarium sp., C. aeruginascens, have shown that the optimum $\mathrm{pH}$ for maximum pigment production varies with the fungal species in submerged fermentation [35,149-152].

Along with chemical parameters, physical parameters such as temperature, light intensity, color of light, agitation speed, and oxygen supply have an impact on pigment production. Studies have also been reported showing the influence of temperature on the biosynthesis of pigments by certain fungal isolates such as M. ruber, T. purpureogenus (formerly known as P. purpurogenum), C. aeruginascens, etc. $[150,152,153]$. Enhancement of yellow pigment production in a Monascus anka mutant strain under submerged fermentation using a two-stage agitation speed control strategy (400 rpm followed by $300 \mathrm{rpm}$ ) has been successfuly reported [154]. A study has also revealed that a sufficient supply of oxygen is necessary for xylindein production by C. aeruginascens [152]. The impact of darkness and different color light on the yield of extracellular and intracellular pigment and biomass has been assessed by various investigators. Most of the studies have shown that incubation in total darkness resulted in enhanced biomass and pigment production [152,155,156]. Studies have also reported that 
there is an enhancement in the pigment production in the case of A. alternata and M. ruber when exposed to blue and red light, respectively [156,157], and in F. oxysporum when exposed to blue and green light [158]. In contrast, reduction in biomass and pigment yield has been observed in I. farinosa, E. nidulans, F. verticillioides, P. purpurogenum (currently known as C. farinosa, A. nidulans, F. fujikuroi, T. purpureogenus, respectively), and M. purpureus when exposed to green and yellow light [155]. Light intensity has also been found to influence the growth and pigment production of $M$. ruber under submerged fermentation [156]. Another study on the influence of moisture content of wood substrate on fungal pigment production in spalted wood was described. Based on the results, low moisture content stimulates the pigmentation in T. versicolor and X. polymorpha, while enhanced pigment production was observed at higher moisture content in the case of I. hispidus, L. brumalis (formerly known as P. brumalis), C. squamosus (formerly known as P. squamosus), and S. cuboideum [34,159]. Optimization of pigment production by simultaneously altering the physical and chemical parameters has been explored by many investigators. Several studies have reported an enhancement of the yield of pigment and biomass from different fungal genera such as Monascus, Penicillium, Fusarium, Alternaria, etc., when the physical and chemical parameters were simultaneously altered [104,133,135,158,160-167].

Nowadays, co-culturing has been found to be an effective method for the activation of cryptic pathways via cell-cell interactions, which ultimately results in the production of novel secondary metabolites such as pigments from the fungi $[168,169]$. Studies have reported that the induction or enhancement in pigment production was possible using co-culturing of fungi with bacteria or yeast, but it was species-specific. In case of Monascus and A. chevalieri, co-culturing was found to be effective, whereas in case of F. oxysporum, the results were negative [158,170]. Co-culturing of C. neoformans with Klebsiella aerogenes led to synthesis of melanin by the fungus, using dopamine synthesized by bacteria [171]. Researchers have also found that many fungi produce different types of zone lines when co-cultured with other fungi. Zone lines are narrow, dark marks composed of pigments (primarily melanin) produced in decaying wood by fungi in response to other fungi, to self-isolate from other decaying fungi and protect their resources [172]. It has been observed that many white rot fungi such as T. versicolor, Stereum gausapatum, Bjerkandera adusta, X. polymorpha, and few brown rot fungi (Poria weirii, Piptoporus betulinus) produce zone lines upon detection of another fungus in their territory [173]. T. versicolor and B. adusta were found to be the best fungal pair which produce zone lines upon co-culturing, whereas X. polymorpha produces zone lines individually in the absence of other fungi [174]. This clearly reveals that the method of co-culturing of these fungi has a significant impact on their pigment production which supplies pigments used for coloring different types of woods in order to enhance their market value.

Various modes of cultivation and various methods and techniques of pigment extraction were investigated by several researchers to enhance fungal pigment production and recovery. Different strategies such as the use of different surfactants (Tween 80, Span 20, Triton X-100, and polyethylene glycerol polymer 8000), different solvents (acetone, acetonitrile, chloroform, cyclohexane, chloramphenicol, dichloromethane, dimethyl sulfoxide, hexane, isooctane, methanol, methyl sulfoxide, pyridine, tetrahydrofuran, and water), and potential extraction techniques (pressurized liquid extraction technique) have also been assessed, compared, and confirmed by researchers for the rapid extraction and enhanced recovery of pigments from submerged fermentation [72,134,175-177]. Researchers also suggested the use of shake culture methods using water as a carrier instead of using wood-based malt-agar media for pigment production from wood-degrading fungi [178].

Genetic engineering techniques for enhanced pigment production in fungi have been reported $[1,20,179]$. Certain genetic approaches such as alteration or modifications of genes, cloning of genes, or elimination of non-essentilal genes (mycotoxins) have been investigated for increasing pigment production and reducing mycotoxins production in fungi [180-182]. The manipulation of biosynthetic pathways has also been investigated by researchers for boosting fungal pigment production. A study on F. graminearum has shown that the transcription factor AurR1 has a positive regulatory effect on the aurofusarin gene cluster, enhancing the production of aurofusarin [183]. 
A recent study on Monascus strains, revealed that transcription factors play an important regulatory role in pigment diversity [184]. More research on this aspect may lead to enhanced pigment production.

\section{Applications or Biological Activities of Fungal Pigments}

Many fungal pigments have been reported to have a variety of biological applications because of their different properties such as antimicrobial, antioxidant, anticancer, and cytotoxic activities in addition to coloring property $[1,20,25,179]$; however, the degree of purity of pigments investigated in the various studies is not always known.

\subsection{Fungal Pigments as Food Colorants}

The majority of work done on fungal pigments is related to their use as food colorants. The possibility of the use of fungal pigments in different industries, particularly in the food industry, has been revealed long ago by many researchers $[9,25,46,48,179,185-187]$. The potential of fungal pigments to be used as food colorants or as food additives in different food products has been assessed by many researchers $[51,188]$. Some of the fungal pigments have already entered into the market as food colorants such as Monascus pigments, arpink red from P. oxalicum, riboflavin from Ashbya gossypii, and $\beta$-carotene from B. trispora $[12,25,189]$.

\subsection{Fungal Pigments as Antimicrobial Agents}

Numerous microbial pigments have been reported to possess many health benefits over synthetic pigments $[8,14]$. Several studies have proved that the pigments or pigment extracts of certain species of fungal genera (Monascus, Fusarium, Talaromyces, Trichoderma, Penicillium, and Aspergillus) and yeast R. glutinis possess antimicrobial activity against different pathogenic bacteria as well as yeast and fungi. All these studies suggest the potential use of bioactive pigments as food preservatives or as antibacterial ingredients in the food and pharmaceutical industries $[19,66,70,82,135,166,189-194]$. Similarly, the antimicrobial potential against selected pathogenic bacteria of different types of fabrics (cotton, silk, etc.) dyed with pigments of fungi (A. alternata and Thermomyces spp.) has also been evaluated, and positive results of these studies suggest their possible use in producing specific products for medical application, such as bandages, suture threads, face masks, etc. [195-197].

\subsection{Fungal Pigments as Antioxidant Agents}

It has been reported that microbial pigments such as carotenoids, violacein, and naphthoquinones have antioxidant potential. Many review articles mention the antioxidant potential of pigments from certain fungi and yeast $[1,17,20,179,198,199]$. Studies on assessment of the antioxidant activity of the pigments of certain fungi such as Penicillium (P. miczynskii, P. purpureogenum, P. purpuroscens, Penicillium sp.), Fusarium sp., Thermomyces sp., Chaetomium sp., Sanghuangporus baumii, Stemphylium lycopersici, and species of Trichoderma (T. afroharzianum, Trichoderma spp.) confirm the promising antioxidant potential and their possible applications in the healthcare industry $[71,97,192,200,201]$.

\subsection{Fungal Pigments as Cytotoxic Agents}

The cytotoxic activity of pigments of certain fungal isolates (F. oxysporum, T. verruculosus, and Chaetomium spp.) has been assessed by many researchers using different methods such as sour orange seeds toxicity assay or yeast toxicity test (YTT) using Saccharomyces cerevisiae, brine shrimp lethality bioassay, or cell counting kit-8 (CCK-8) assay. These studies confirm the possible application of pigments in different industries, especially in health and pharmaceutical ones $[47,82,106,202]$. A latest study on the evaluation of dermal toxicity of pigments of Thermomyces spp. and P. purpurogenum in Wistar rats has revealed the nontoxic nature of pigments and suggested its potential application in cosmetics and dyeing [203]. 


\subsection{Fungal Pigments as Anticancer Agents}

Fungal pigments are known to possess anticancer/antitumor activity. Several studies have revealed the fungal pigments as a potential anticancer drug. Pigments of Monascus species (M. purpureus and M. pilosus) such as monascin, ankaflavin, monaphilone $\mathrm{A}-\mathrm{B}$, monasphilone $\mathrm{A}-\mathrm{B}$, monapilol $\mathrm{A}-\mathrm{D}$, and monapurone $\mathrm{A}-\mathrm{C}$ have been proved to possess anticancer/antitumor potential against different types of cancers, such as mouse skin carcinoma, human laryngeal carcinoma, human colon adenocarcinoma, human hepatocellular carcinoma, and pulmonary adenocarcinoma (Figure 12) [32,56-58,204,205]. Besides Monascus, pigments from other fungi such as norsolorinic acid from A. nidulans, shiraiarin from Shiraia bambusicola, alterporriol K, alterporriol L, and alterporriol M from Alternaria spp., benzoquinone from Fusarium spp., and an uncharacterized red pigment from $F$. chlamydosporum have also been reported to have anticancer, antitumor, or antiproliferative activity mainly against human breast cancer cell lines (MCF-7, MDA-MB-435, and MCF-7 b), whereas hypocrellin D from S. bambusicola shows anticancer activity against other cancer cell lines (Bel-7721, A-549, and Anip-973) (Figure 12) [62,68,88,89,113].

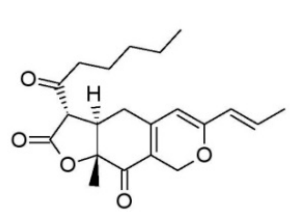

Monascin

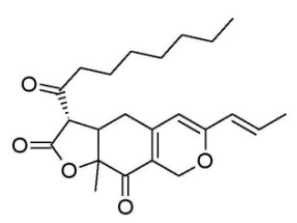

Ankaflavin

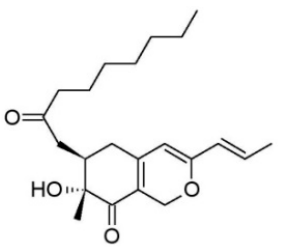

Monasphilone A

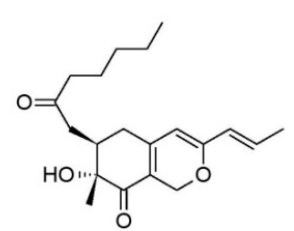

Monasphilone B
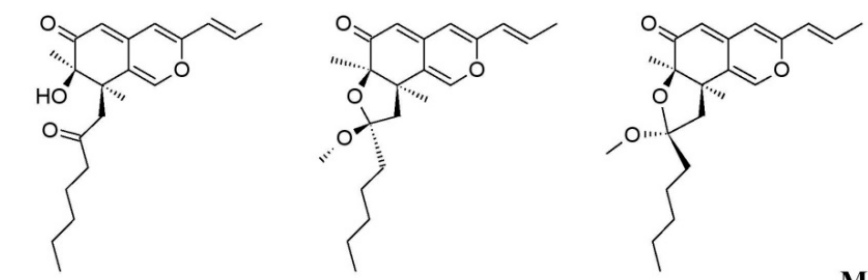<smiles>[R]C(=O)C1=C2C=C3C=C(C=CC)OC=C3C(O)C2(C)OC1=O</smiles>

Monapurone A

Monapurone B

Monapurone C

Monophilol A $\left(\mathrm{R}=-\mathrm{C}_{7} \mathrm{H}_{15}\right)$

Monophilol B $\left(\mathrm{R}=-\mathrm{C}_{5} \mathrm{H}_{11}\right)$<smiles>[R]C(=O)C1=C2C=C3C=C(C=CC)OC=C3[C@@](C)(CC(C)=O)[C@@]2(C)OC1=O</smiles>

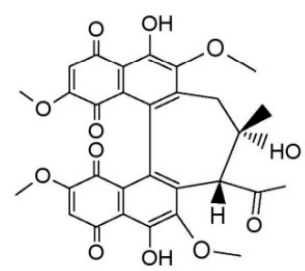

Monophilol C $\left(\mathbf{R}=-\mathrm{C}_{7} \mathrm{H}_{15}\right)$

Monophilol D $\left(\mathrm{R}=-\mathrm{C}_{5} \mathrm{H}_{11}\right)$<smiles>CCCCCC(=O)c1c(O)cc2c(c1O)C(=O)c1c(O)cc(O)cc1C2=O</smiles>

Hypocrellin D<smiles>COc1cc(O)c2c(c1)C(=O)c1c(O)cc(C)cc1C2=O</smiles>

Shiraiarin<smiles>COC1=C(O)C(=O)c2c(O)c3c(c(O)c2C1OC)C(OC)C(O)C(O)C3O</smiles>

Benzoquinone<smiles></smiles>

Alterporriol K

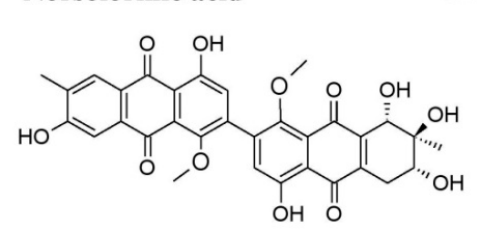

Alterporriol L

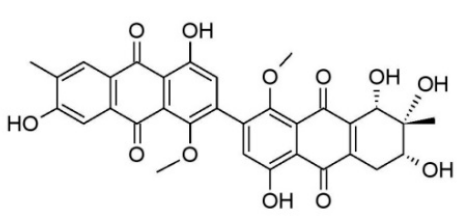

Alterporriol M

Figure 12. Pigments from different taxonomic groups of fungi having promising anticancer or antitumor potential, re-drawn from $[32,56-58,62,68,88,89,113,204,205]$. 


\subsection{Fungal Pigments in the Cosmetic Industry}

As the demand for natural products is increasing in the market, cosmetic industries are also in search of new types of natural pigments to replace synthetic pigments. Among the natural pigments, the use of fungal pigments is also rapidly expanding in cosmetics because of their advantages. Fungal pigments, especially melanin, carotenoids, lycopene, etc., have been reported for their application in cosmetics, sunscreens, sun lotions, sunblocks, face creams, anti-aging facials, etc. [1,206,207]. Excitingly, some of the fungal pigments (Monascus pigments and Monascus-like pigments) have already entered the market for their application in cosmetics such as skin conditioning and skin care products, lipsticks, etc. [25].

\subsection{Fungal Pigments in the Textile Industry}

The textile industry is the largest industry after agriculture in terms of economic contribution and employment generation. It majorly depends on synthetic dyes for dyeing different types of fabrics (cotton, silk, and wool). Currently, natural pigments from fungi, with their many advantages (eco-friendly, non-toxic, easy degradation, high colorfastness, high staining capability, etc.) over hazardous synthetic pigments, have proven to be a good alternative to the synthetic dyes in the textile industry. Many investigations have shown that organic pigments produced by fungi have extensive applications in the textile industry $[1,5,8,18,25,207]$.

The literature reveals that only a handful of studies have investigated the application of fungal pigments in the textile industry, especially for dyeing different types of fabrics, such as cotton, silk, and wool. Various studies on the dyeing potential of pigments of different species of fungal genera (Monascus, Fusarium, Aspergillus, Penicillium, Talaromyces, Trichoderma, Alternaria, Curvularia, Chlorociboria, Scytalidium, Cordyceps, Acrostalagmus, Bisporomyces, Cunninghamella, Thermomyces, and Phymatotrichum) for different types of fabrics such as wool, cotton yarn, silk, polyester, and nylon have been reported [37,42,47,106,108,124,195,196,208-211]. Studies on the dyeing potential of pigments from wood spalting fungi (red pigment from S. cuboideum, yellow pigment from S. ganodermophthorum, and green pigment $C$. aeruginosa) have shown the possible use of these pigments for deying bleached cotton, spun polyacrylic, spun polyamide (nylon 6.6), worsted wool, spun polyester (Dacron 54), and garment fabrics, because of their high stability and good colorfastness to washing $[37,212]$. Another study has revealed that natural oils cannot be used in conjunction with these fungal pigments, as these fungal pigments are unstable in natural oils [42]. Results of all these studies have shown that these fungal pigments have good color stability, colorfastness properties, and dye uptake potential. Moreover, these fungal pigments do not have any adverse effects on fabric and are non-toxic to human skin. Therefore, the scope of applications of fungal pigments has the opportunity to expand into the textile and clothing industry.

\subsection{Fungal Pigments in Dyeing Woods or as Color Modifiers}

Pigment produced by wood-decaying fungi such as T. versicolor, X. polymorpha, I. hispidus, S. cuboideum, B. adusta, C. aeruginascens, and Arthrographis cuboidea have been used for dyeing different types of wood samples to increase their commercial importance $[173,174,213]$. Researchers have successfully used the red, green, and yellow pigments obtained from S. cuboideum, S. ganodermophthorum, and C. aeruginosa, respectively, to attenuate the presence of blue stain on wood samples of Pinus spp. [39].

\subsection{Fungal Pigments in (Opto) Electronics}

A recent study of the (opto)electronic properties of blends of the pigment xylindein extracted from C. aeruginosa has revelaed that this pigment has high photostability and electron mobility in amorphous films, which suggests its possible use for the development of sustainable, organic semiconductor materials [214,215]. 


\section{Conclusions}

Several advantages of fungal pigments over synthetic pigments have increased the demand for fungal pigments worldwide in recent years. This increased public awareness, eco-safety, and health concerns as well as the application of strict environmental and ecological rules and regulations, have challenged researchers to undertake both qualitative and quantitative research on pigments derived from clean, eco-friendly bio-resources, such as fungi, having minimal ecological negative impacts. Therefore, there is a necessity to explore other novel, safe pigments from the diverse taxonomic group of fungi, to meet the existing demand of eco-friendly pigments. Though several fungal strains are known as pigment producers, a large number of fungi have not been systematically explored for their pigment-producing capability. Therefore, there is a great need to explore the vast fungal diversity for rare, novel, safe pigments, using appropriate tools and techniques. A review of the literature revealed that most of the studies focused on the application of fungal pigments in the food and healthcare industries; however, fungal pigments need to pass toxicity tests and quality tests and receive many regulatory approvals before their final entry into the market as food colorants or as drugs. Therefore, the application of fungal pigments in these areas is quite difficult.

Moreover, meager studies on the applicability of fungal pigments in other areas such as textiles, paints, varnishes, and daily household utensils leave immense possibilities to explore the indigenous diversity of fungi for their pigment production potential and their applications in different sectors, including the textile industry. In addition to the coloring properties, the biological properties of fungal pigments may open new avenues for their use in the production of valuable textiles for medical use. This provides an extensive area of exploration to identify natural, eco-friendly fungal pigments and develop their diverse applications to satisfy the public interest and market demand.

Author Contributions: Writing-original draft preparation, A.C.L.; writing-review and editing, S.K.S., L.D., P.N.S.

Funding: This research received no external funding.

Acknowledgments: We thank Prashant Dhakephalkar, Director, MACS' Agharkar Research Institute, Pune, for encouragement and providing necessary facilities to carry out the research work. Ajay C. Lagashetti acknowledges CSIR (Council of Scientific and Industrial Research), New Delhi, for granting Junior Research Fellowship (JRF) and S. P. Pune University, Pune, for granting permission to register for Ph.D. degree. Ajay C. Lagashetti also thanks Siddharam Bagale for his major help in re-drawing chemical structures in manuscript. Laurent Dufossé deeply thanks the Conseil Régional de La Réunion, Réunion island, Indian Ocean, for continuous financial support of research activities dedicated to microbial pigments.

Conflicts of Interest: The authors declare no conflict of interest.

\section{References}

1. Rao, M.P.N.; Xiao, M.; Li, W.J. Fungal and bacterial pigments: Secondary metabolites with wide application. Front. Microbiol. 2017, 8, 1113.

2. Downham, A.; Collins, P. Coloring our foods in the last and next millennium. Int. J. Food Sci. Technol. 2000, 35, 5-22. [CrossRef]

3. Osman, M.Y.; Sharaf, I.A.; Osman, H.M.Y.; El-Khouly, Z.A.; Ahmed, E.I. Synthetic organic food coloring agents and their degraded products: Effects on human and rat cholinesterases. Br. J. Biomed. Sci. 2004, 61, 128-132. [CrossRef] [PubMed]

4. Babitha, S. Microbial pigments. In Biotechnology for Agro-Industrial Residues Utilization; Nigam, P.S., Pandey, A., Eds.; Springer: Dordrecht, The Netherlands, 2009; pp. 147-162.

5. Samanta, A.K.; Agarwal, P. Application of natural dyes on textiles. Indian J. Fibre Text. Res. 2009, 34, $384-399$.

6. Ratna, P.B.S. Pollution due to synthetic dyes toxicity and carcinogenicity studies and remediation. Int. J. Environ. Sci. 2012, 3, 940-955.

7. Arora, S. Textile dyes: Its impact on the environment and its treatment. J. Bioremediat. Biodegrad. $2014,5,1$. [CrossRef]

8. Akilandeswari, P.; Pradeep, B.V. Exploration of industrially important pigments from soil fungi. Appl. Microbiol. Biotechnol. 2016, 100, 1631-1643. [CrossRef] 
9. Chattopadhyay, P.; Chatterjee, S.; Sen, S.K. Biotechnological potential of natural food grade biocolorants. Afr. J. Biotechnol. 2008, 7, 2972-2985.

10. Joshi, V.K.; Attri, D.; Bala, A.; Bhushan, S. Microbial pigments. Indian J. Biotechnol. 2003, 2, 362-369.

11. Aberoumand, A. A review article on edible pigments properties and sources as natural biocolorants in foodstuff and food industry. World J. Dairy Food Sci. 2011, 6, 71-78.

12. Dufossé, L. Microbial production of food grade pigments. Food Technol. Biotechnol. 2006, 44, 313-321.

13. Latha, B.V.; Jeevaratnam, K. Purification and characterization of the pigments from Rhodotorula glutinis DFR-PDY isolated from a natural source. Glob. J. Biotechnol. Biochem. 2010, 5, 166-174.

14. Nagpal, N.; Munjal, N.; Chatterjee, S. Microbial pigments with health benefits-A mini review. Trends Biosci. 2011, 4, 157-160.

15. Ahmad, W.A.; Ahmad, W.Y.W.; Zakaria, Z.A.; Yusof, N.Z. Isolation of pigment-producing bacteria and characterization of the extracted pigments. In Application of Bacterial Pigments as a Colorant; SpringerBriefs in Molecular Science; Springer: Berlin/Heidelberg, Germany, 2012; pp. 25-44.

16. Kirti, K.; Amita, S.; Priti, S.; Kumar, A.M.; Jyoti, S. Colorful world of microbes: Carotenoids and their applications. Adv. Biol. 2014, 1, 1-13. [CrossRef]

17. Mata-Gomez, L.C.; Montanez, J.C.; Mendez-Zavala, A.; Aguilar, C.N. Biotechnological production of carotenoids by yeasts: An overview. Microb. Cell Fact. 2014, 13, 12. [CrossRef]

18. Kumar, A.; Vishwakarma, H.S.; Singh, J.; Dwivedi, S.; Kumar, M. Microbial pigments: Production and their applications in various industries. Int. J. Phram. Chem. Biol. Sci. 2015, 5, 203-212.

19. Sarkar, S.L.; Saha, P.; Sultana, N.; Akter, S. Exploring textile dye from microorganisms, an eco-friendly alternative. Microbiol. Res. J. Int. 2017, 18, 1-9. [CrossRef]

20. Ramesh, C.; Vinithkumar, N.V.; Kirubagaran, R.; Venil, C.K.; Dufossé, L. Multifaceted applications of microbial pigments: Current knowledge, challenges and future directions for public health implications. Microorganisms 2019, 7, 186. [CrossRef]

21. Venil, C.K.; Zakaria, Z.A.; Ahmad, W.A. Bacterial pigments and their applications. Process. Biochem. 2013, 48, 1065-1079. [CrossRef]

22. Gupta, C.; Prakash, D.; Gupta, S. Natural useful therapeutic products from microbes. J. Microbiol. Exp. 2014, 1, 30-37. [CrossRef]

23. Numan, M.; Bashir, S.; Mumtaz, R.; Tayyab, S.; Rehman, N.U.; Khan, A.L.; Shinwari, Z.K.; Al-Harrasi, A. Therapeutic applications of bacterial pigments: A review of current status and future opportunities. 3 Biotech 2018, 8, 207. [CrossRef]

24. Indra Arulselvi, P.; Umamaheswari, S.; Ranandkumar Sharma, G.; Karthik, C.; Jayakrishna, C. Screening of yellow pigment producing bacterial isolates from various eco-climatic areas and analysis of the carotenoid produced by the isolate. J. Food Process. Technol. 2014, 5, 292.

25. Caro, Y.; Venkatachalam, M.; Lebeau, J.; Fouillaud, M.; Dufossé, L. Pigments and colorants from filamentous fungi. In Fungal Metabolites; Merillon, J.-M., Ramawat, K.G., Eds.; Springer International Publishing: Cham, Switzerland, 2017; pp. 499-568.

26. Gmoser, R.; Ferreira, J.A.; Lennartsson, P.R.; Taherzadeh, M.J. Filamentous ascomycetes fungi as a source of natural pigments. Fungal Biol. Biotechnol. 2017, 4, 4. [CrossRef] [PubMed]

27. Manikprabhu, D.; Lingappa, K. $\gamma$ Actinorhodin a natural and attorney source for the synthetic dye to detect acid production of fungi. Saudi J. Biol. Sci. 2013, 20, 163-168. [CrossRef] [PubMed]

28. Blanchette, R.A.; Wilmering, A.M.; Baumeister, M. The use of green-stained wood caused by the fungus Chlorociboria in intarsia masterpieces from the 15th century. Holzforsch Int. J. Biol. Chem. Phys. Technol. Wood 1992, 46, 225-232.

29. Butler, M.J.; Day, A.W. Fungal melanins: A review. Can. J. Microbiol. 1998, 44, 1115-1136. [CrossRef]

30. Sakaki, T.; Shibata, M.; Mukai, K.; Sakai, M.; Wakamatsu, K.; Miyauchi, S. Chlorociboria aeruginosa pigment as algicide. Jpn. Kokai Tokkyo Koho JP 2002, 2002291493.

31. Carvalho, J.C.; Pandey, A.; Babitha, S.; Soccol, C.R. Production of Monascus biopigments: An overview. Agro Food Ind. Hi-Tech 2003, 14, 37-42.

32. Feng, Y.; Shao, Y.; Chen, F. Monascus pigments. J. Appl. Microbiol. Biotechnol. 2012, 96, 1421-1440. [CrossRef]

33. Robinson, S.C.; Tudor, D.; Snider, H.; Cooper, P.A. Stimulating growth and xylindein production of Chlorociboria aeruginascens in agar-based systems. AMB Express 2012, 2, 15. [CrossRef] 
34. Tudor, D. Fungal Pigment Formation in Wood Substrate. Ph.D. Thesis, University of Toronto, Toronto, ON, Canada, 2013.

35. Tudor, D.; Robinson, S.C.; Cooper, P.A. The influence of $\mathrm{pH}$ on pigment formation by lignicolous fungi. Int. Biodeterior. Biodegrad. 2013, 80, 22-28. [CrossRef]

36. Robinson, S.C.; Tudor, D.; Zhang, W.R.; Ng, S.; Cooper, P.A. Ability of three yellow pigment producing fungi to colour wood under controlled conditions. Int. Wood Prod. J. 2014, 5, 103-107. [CrossRef]

37. Hinsch, E.M.; Chen, H.L.; Weber, G.; Robinson, S.C. Colorfastness of extracted wood-staining fungal pigments on fabrics: A new potential for textile dyes. J. Text. Appar. Technol. Manag. 2015, 9, 1-11.

38. Tam, W.T.E.; Tsang, C.C.; Lau, K.P.S.; Woo, C.Y.P. Polyketides, toxins, and pigments in Penicillium marneffei. Toxins 2015, 7, 4421-4436. [CrossRef] [PubMed]

39. Hernandez, V.A.; Galleguillos, F.; Robinson, S. Fungal pigments from spalting fungi attenuating blue stain in Pinus spp. Int. Biodeterior. Biodegrad. 2016, 107, 154-157. [CrossRef]

40. Robinson, S.C.; Michaelsen, H.; Robinson, J.C. Spalted Wood, History, Science and Art of an Unique Material; Schiffer Publishing: Atglen, PA, USA, 2016; pp. 1-288.

41. Souza, P.N.C.; Grigoletto, T.L.B.; Moraes, L.A.B.; Abreu, L.M.; Souza, L.H.; Santos, C.; Galvao, L.R.; Cardoso, P.G. Production and chemical characterization of pigments in filamentous fungi. Microbiology 2016, 162, 12-22. [CrossRef] [PubMed]

42. Agurto, M.E.P.; Gutierrez, S.M.V.; Chen, H.-L.; Robinson, S.C. Wood-rotting fungal pigments as colorant coatings on oil-based textile dyes. Coatings 2017, 7, 152. [CrossRef]

43. Avalos, J.; Pardo-Medina, J.; Parra-Rivero, O.; Ruger-Herreros, M.; Rodriguez-Ortiz, R.; Hornero-Mendez, D.; Limon, M.C. Carotenoid biosynthesis in Fusarium. J. Fungi 2017, 3, 39. [CrossRef]

44. Pombeiro-Sponchiado, S.R.; Sousa, G.S.; Andrade, J.C.; Lisboa, H.F.; Gonçalves, R.C. Production of melanin pigment by fungi and its biotechnological applications. In Melanin; Blumenberg, M., Ed.; InTechOpen: London, UK, 2017; pp. 45-75.

45. Vega Gutierrez, P.; Robinson, S. Determining the presence of spalted wood in spanish marquetry woodworks of the 1500s through the 1800s. Coatings 2017, 7, 188. [CrossRef]

46. Mortensen, A. Carotenoids and other pigments as natural colorants. Pure Appl. Chem. 2006, 78, 1477-1491. [CrossRef]

47. Nagia, F.A.; El-Mohamedy, R.S.R. Dyeing of wool with natural anthraquinone dyes from Fusarium oxysporum. Dyes Pigm. 2007, 75, 550-555. [CrossRef]

48. Mapari, S.A.S.; Thrane, A.U.; Meyer, A.S. Fungal polyketide azaphilone pigments as future natural food colorants? Trends Biotechnol. 2010, 28, 300-307. [CrossRef] [PubMed]

49. Dufossé, L.; Fouillaud, M.; Caro, Y.; Mapari, S.A.S.; Sutthiwong, N. Filamentous fungi are large-scale producers of pigments and colorants for the food industry. Curr. Opin. Biotechnol. 2014, 26, 56-61. [CrossRef] [PubMed]

50. Went, F.A.F.C. Monascus purpureus le champignon de l'ang-quac une nouvelle thelebolee. Ann. Des. Sci. Nat. Bot. Biol. Veg. 1895, 8, 1-18.

51. Fabre, C.E.; Santerre, A.L.; Loret, M.O.; Baberian, R.; Pareilleux, A.; Goma, G.; Blanc, P.J. Production and food applications of the red pigments of Monascus ruber. J. Food Sci. 1993, 58, 1099-1102. [CrossRef]

52. Hajjaj, H.; Klaebe, A.; Loret, M.O.; Tzedakis, T.; Goma, G.; Blanc, P.J. Production and identification of $\mathrm{N}$-glucosylrubropunctamine and $\mathrm{N}$-glucosylmonascorubramine from Monascus ruber and occurrence of electron donor-acceptor complexes in these red pigments. Appl. Environ. Microbiol. 1997, 63, 2671-2678.

53. Lian, X.; Wang, C.; Guo, K. Identification of new red pigments produced by Monascus ruber. Dyes Pigm. 2007, 73, 121-125. [CrossRef]

54. Loret, M.O.; Morel, S. Isolation and structural characterization of two new metabolites from Monascus. J. Agric. Food Chem. 2010, 58, 1800-1803. [CrossRef]

55. Chen, W.; Chen, R.; Liu, O.; He, Y.; He, K.; Ding, X.; Kang, L.; Guo, X.; Xie, N.; Zhou, Y.; et al. Orange, red, yellow: Biosynthesis of azaphilone pigments in Monascus fungi. Chem. Sci. 2017, 8, 4917-4925. [CrossRef]

56. Hsu, Y.-W.; Hsu, L.-C.; Liang, Y.-H.; Kuo, Y.-H.; Pan, T.-M. Monaphilones A-C, three new antiproliferative azaphilone derivatives from Monascus purpureus NTU 568. J. Agric. Food Chem. 2010, 58, 8211-8216. [CrossRef]

57. Li, J.-J.; Shang, X.-Y.; Li, L.-L.; Liu, M.-T.; Zheng, J.-Q.; Jin, Z.L. New cytotoxic azaphilones from Monascus purpureus-fermented rice (red yeast rice). Molecules 2010, 15, 1958-1966. [CrossRef] [PubMed] 
58. Hsu, Y.-W.; Hsu, L.-C.; Liang, Y.-H.; Kuo, Y.-H.; Pan, T.-M. New bioactive orange pigments with yellow fluorescence from Monascus-fermented Dioscorea. J. Agric. Food Chem. 2011, 59, 4512-4518. [CrossRef] [PubMed]

59. Mukherjee, G.; Singh, S.K. Purification and characterization of a new red pigment from Monascus purpureus in submerged fermentation. Process Biochem. 2011, 46, 188-192. [CrossRef]

60. Kaur, B.; Chakraborty, D.; Kaur, H. Production and evaluation of physicochemical properties of red pigment from Monascus purpureus MTCC 410. Internet J. Microbiol. 2008, 7, 1-6.

61. Mapari, S.A.S.; Meyer, A.S.; Thrane, U.; Frisvad, J.C. Identification of potentially safe promising fungal cell factories for the production of polyketide natural food colorants using chemotaxonomic rationale. Microb. Cell Fact. 2009, 8, 24. [CrossRef]

62. Soumya, K.; Narasimha Murthy, K.; Sreelatha, G.L.; Tirumale, S. Characterization of a red pigment from Fusarium chlamydosporum exhibiting selective cytotoxicity against human breast cancer MCF-7 cell lines. J. Appl. Microbiol. 2018, 125, 148-158. [CrossRef]

63. Steyn, P.S.; Wessels, P.L.; Marasas, W.F.O. Pigments from Fusarium moniliforme Shledon: Structure and ${ }^{13} \mathrm{C}$ nuclear magnetic resonance assignments of an azaanthraquinone and three naphthoquinones. Tetrahedron 1979, 35, 1551-1555. [CrossRef]

64. Medenstev, A.G.; Arinbasarova, A.; Akimenko, V.K. Biosynthesis of naphthoquinone pigments by fungi of the genus. Fusarium. Prikl. Biokhim. Mikrobiol. 2005, 41, 573-577.

65. Pradeep, F.S.; Palaniswamya, M.; Ravib, S.; Thangamanib, A.; Pradeep, B.V. Larvicidal activity of a novel isoquinoline type pigment from Fusarium moniliforme KUMBF1201 against Aedes aegypti and Anopheles stephensi. Process Biochem. 2015, 50, 1479-1486. [CrossRef]

66. Frandsen, R.J.N.; Rasmussen, S.A.; Knudsen, P.B.; Uhlig, S.; Petersen, D.; Lysoe, E.; Gotfredsen, C.H.; Giese, H.; Larsen, T.O. Black perithecial pigmentation in Fusarium species is due to the accumulation of 5-deoxybostrycoidin-based melanin. Sci. Rep. 2016, 6, 26206. [CrossRef]

67. Lebeau, J.; Petit, T.; Clerc, P.; Dufossé, L.; Caro, Y. Isolation of two novel purple naphthoquinone pigments concomitant with the bioactive red bikaverin and derivates thereof produced by Fusarium oxysporum. Biotechnol. Prog. 2019, 35, e2738. [CrossRef] [PubMed]

68. Zheng, L.; Cai, Y.; Zhou, L.; Huang, P.; Ren, X.; Zuo, A.; Meng, X.; Xu, M.; Liao, X. Benzoquinone from Fusarium pigment inhibits the proliferation of estrogen receptor-positive MCF-7 cells through the NF- $\mathrm{KB}$ pathway via estrogen receptor signalling. Int. J. Mol. Med. 2017, 39, 39-46. [CrossRef] [PubMed]

69. Trisuwan, K.; Khamthong, N.; Rukachaisirikul, V.; Phongpaichit, S.; Preedanon, S.; Sakayaroj, J. Anthraquinone, cyclopentanone, and naphthoquinone derivatives from the sea fan-derived fungi Fusarium spp. PSU-F14 and PSU-F135. J. Nat. Prod. 2010, 73, 1507-1511. [CrossRef] [PubMed]

70. Sibero, M.T.; Triningsih, D.W.; Radjasa, O.K.; Sabdono, A.; Trianto, A. Evaluation of antimicrobial activity and identification of yellow pigmented marine sponge-associated fungi from Teluk Awur, Jepara, Central Java. Indones. J. Biotechnol. 2016, 21, 1-11. [CrossRef]

71. Heo, Y.M.; Kim, K.; Kwon, S.L.; Na, J.; Lee, H.; Jang, S.; Kim, C.H.; Jung, J.; Kim, J.J. Investigation of filamentous fungi producing safe, functional water-soluble pigments. Mycobiology 2018, 46, 269-277. [CrossRef]

72. Lebeau, J.; Venkatachalam, M.; Fouillaud, M.; Petit, T.; Vinale, F.; Dufossé, L.; Caro, Y. Production and new extraction method of polyketide red pigments produced by ascomycetous fungi from terrestrial and marine habitats. J. Fungi 2017, 3, 34. [CrossRef]

73. Velmurugan, P.; Lee, Y.H.; Nanthakumar, K.; Kamala-Kannan, S.; Dufossé, L.; Mapari, S.A.S.; Oh, B.T. Water-soluble red pigments from Isaria farinosa and structural characterization of the main colored component. J. Basic Microbiol. 2010, 50, 581-590. [CrossRef]

74. Wat, C.-K.; Mcinnes, A.G.; Smith, D.G.; Wright, J.L.C.; Vining, L.C. The yellow pigments of Beauveria species. Structures of tenellin and bassianin. Can. J. Chem. 1977, 55, 4090-4098. [CrossRef]

75. Isaka, M.; Chinthanom, P.; Supothina, S.; Tobwor, P. Pyridone and tetramic acid alkaloids from the spider pathogenic fungus Torrubiella sp. BCC 2165. J. Nat. Prod. 2010, 73, 2057-2060. [CrossRef]

76. Devi, S.; Kumar, H.A.K.; Ramachandran, G.; Subramanian, C.; Karuppan, P. Growth and mass spectrometry profile of Alternaria alternata pigment grown in maize grain extract. J. Microbiol. Biotechnol. Food Sci. 2014, 4, 179-184. [CrossRef]

77. Suemitsu, R.; Nakamura, A.; Isono, F.; Sano, T. Isolation and identification of Dactylariol from the culture liquid of Alternaria porri (Ellis) Ciferri. Agric. Biol. Chem. 1982, 46, 1693-1694. [CrossRef] 
78. Okuno, T.; Natsume, I.; Sawai, K.; Sawamura, K.; Furusaki, A.; Matsumoto, T. Structure of antifungal and phytotoxic pigments produced by Alternaria sps. Tetrahedron Lett. 1983, 24, 5653-5656. [CrossRef]

79. Huang, C.H.; Pan, J.H.; Chen, B.; Yu, M.; Huang, H.B.; Zhu, X.; Lu, Y.J.; She, Z.G.; Lin, Y.C. Three bianthraquinone derivatives from the mangrove endophytic fungus Alternaria sp. ZJ9-6B from the South China Sea. Mar. Drugs 2011, 9, 832-843. [CrossRef] [PubMed]

80. Williamson, P.R.; Wakamatsu, K.; Ito, S. Melanin biosynthesis in Cryptococcus neoformans. J. Bacteriol. 1998, 180, 1570-1572.

81. Harki, E.; Talou, T.; Dargent, R. Purification, characterization and analysis of melanin extracted from Tuber melanosporum vitt. Food Chem. 1997, 58, 69-73. [CrossRef]

82. Wang, W.; Liao, Y.; Chen, R.; Hou, Y.; Ke, W.; Zhang, B.; Gao, M.; Shao, Z.; Chen, J.; Li, F. Chlorinated azaphilone pigments with antimicrobial and cytotoxic activities isolated from the deep sea-derived fungus Chaetomium sp. NA-S01-R1. Mar. Drugs 2018, 16, 61. [CrossRef]

83. Suryanarayanan, T.S.; Ravishankar, J.P.; Venkatesan, G.; Murali, T.S. Characterization of the melanin pigment of a cosmopolitan fungal endophyte. Mycol. Res. 2004, 108, 974-978. [CrossRef]

84. O'Leary, M.A.; Hanson, J.R.; Yeoh, B.L. The structure and biosynthesis of hinnuliquinone, a pigment from Nodulisporium hinnuleum. J. Chem. Soc. Perkin Trans. 1 1984, 1, 567-569. [CrossRef]

85. Wang, L.; Dong, J.Y.; Song, H.C.; Shen, K.Z.; Wang, L.M.; Sun, R.; Wang, C.R.; Gao, Y.X.; Li, G.H.; Li, L.; et al. Three new naphthoquinone pigments isolated from the freshwater fungus, Astrosphaeriella papuana. Planta Med. 2009, 75, 1339-1343. [CrossRef]

86. Duarte, A.W.F.; de Menezes, G.C.A.; e Silva, T.R.; Bicas, J.L.; Oliveira, V.M.; Rosa, L.H. Antarctic fungi as producers of pigments. In Fungi of Antarctica; Rosa, L., Ed.; Springer: Cham, Switzerland, 2019; pp. $305-318$.

87. Singh, S.M.; Singh, P.N.; Singh, S.K.; Sharma, P.K. Pigment, fatty acid and extracellular enzyme analysis of a fungal strain Thelebolus microsporus from Larsemann Hills, Antarctica. Polar Rec. 2014, 50, 31-36. [CrossRef]

88. Fang, L.Z.; Qing, C.; Shao, H.J.; Yang, Y.D.; Dong, Z.J.; Wang, F.; Zhao, W.; Yang, W.Q.; Liu, J.K. Hypocrellin $\mathrm{D}$, a cytotoxic fungal pigment from fruiting bodies of the ascomycete Shiraia bambusicola. J. Antibiot. 2006, 59, 351-354. [CrossRef] [PubMed]

89. Cai, Y.; Ding, Y.; Tao, G.; Liao, X. Production of 1, 5-dihydroxy-3-methoxy-7-methylanthracene-9, 10-dione by submerged culture of Shiraia bambusicola. J. Microbiol. Biotechnol. 2008, 18, 322-327. [PubMed]

90. Avalos, J.; Prado-Cabrero, A.; Estrada, A.F. Neurosporaxanthin production by Neurospora and Fusarium. In Microbial Carotenoids from Fungi: Methods and Protocols; Barredo, J.-L., Ed.; Springer Protocols: Totowa, NJ, USA, 2012; pp. 263-274.

91. Teixeira, M.F.S.; Martins, M.S.; Da Silva, J.C.; Kirsch, L.S.; Fernandes, O.C.C.; Carneiro, A.L.B.; De Conti, R.; Durrn, N. Amazonian biodiversity: Pigments from Aspergillus and Penicillium-characterizations, antibacterial activities and their toxicities. Curr. Trends Biotechnol. Pharm. 2012, 6, 300-311.

92. Viggiano, A.; Salo, O.; Ali, H.; Szymanski, W.; Lankhorst, P.P.; Nygard, Y.; Bovenberg, R.A.L.; Driessena, A.J.M. Pathway for the biosynthesis of the pigment Chrysogine by Penicillium chrysogenum. Appl. Environ. Microbiol. 2018, 84, 1-11. [CrossRef] [PubMed]

93. Suzuki, S.; Hosoe, T.; Nozawa, K.; Kawai, K.; Yaguchi, T.; Udagawa, S. Antifungal substances against pathogenic fungi, Talaroconvolutins, from Talaromyces convolutes. J. Nat. Prod. 2000, 63, 768-772. [CrossRef]

94. Santos, P.O.; Ferraz, C.G.; Soares, A.C.F.; Miranda, F.M.; da Silva, F.; de Abreu Roque, M.R. Sclerotiorin, a novel pigment from Penicillium mallochii. In Proceedings of the 6th Brazilian Conference on Natural Products, Federal University of Espirito Santo Victoria, Vitoria, Brazil, 5-8 November 2017.

95. Ogihara, J.; Kato, J.; Oishi, K.; Fujimoto, Y. PP-R, 7-(2-Hydroxyethyl)-Monascorubramine, a red pigment produced in the mycelia of Penicillium sp. AZ. J. Biosci. Bioeng. 2001, 91, 44-47. [CrossRef]

96. Pandey, N.; Jain, R.; Pandey, A.; Tamta, S. Optimisation and characterization of the orange pigment produced by a cold-adapted strain of Penicillium sp. (GBPI_P155) isolated from mountain ecosystem. Mycology 2018, 9, 81-92. [CrossRef]

97. Dhale, M.A.; Vijay-Raj, A.S. Pigment and amylase production in Penicillium sp. NIOM-02 and its radical scavenging activity. Int. J. Food Sci. Technol. 2009, 44, 2424-2430. [CrossRef]

98. Jiang, Y.; Li, H.B.; Chen, F.; Hyde, K.D. Production potential of water-soluble Monascus red pigment by a newly isolated Penicillium sp. J. Agric. Technol. 2005, 1, 113-126.

99. Chintapenta, L.K.; Rath, C.C.; Maringinti, B.; Ozbay, G. Pigment production from a mangrove Penicillium. Afr. J. Biotechnol. 2014, 13, 2668-2674. 
100. Frisvad, J.C.; Yilmaz, N.; Thrane, U.; Rasmussen, K.B.; Houbraken, J.; Samson, R.A. Talaromyces atroroseus, a new species efficiently producing industrially relevant red pigments. PLoS ONE 2013, 8, e84102. [CrossRef] [PubMed]

101. Bhardwaj, S.; Shukla, A.; Mukherjee, S.; Sharma, S.; Guptasarma, P.; Chakraborti, A.K.; Chakrabarti, A. Putative structure and characteristics of red water-soluble pigment secreted by Penicillium marneffei. Med. Mycol. 2007, 45, 419-427. [CrossRef] [PubMed]

102. Mendez-Zavala, A.; Contreras-Esquivel, J.C.; Lara-Victoriano, F.; Rodriguez-Herrera, R.; Aguilar, C.N. Fungal production of the red pigment using a xerophilic strain Penicillium purpurogenum GH-2. Rev. Mex. Ing. Quim. 2007, 6, 267-273.

103. Arai, T.; Koganei, K.; Umemura, S.; Kojima, R.; Kato, J.; Kasumi, T.; Ogihara, J. Importance of the ammonia assimilation by Penicillium purpurogenum in amino derivative Monascus pigment, PP-V production. AMB Express 2013, 3, 19. [CrossRef]

104. Sethi, B.K.; Parida, P.; Sahoo, S.L.; Dikshit, B.; Pradhan, C.; Sena, S.; Behera, B.C. Extracellular production and characterization of red pigment from Penicillium purpurogenum BKS9. Alger. J. Nat. Prod. 2016, 4, 379-392.

105. Ogbonna, C.N.; Aoyagi, H.; Ogbonna, J.C. Isolation and identification of Talaromyces purpurogenus and preliminary studies on its pigment production potentials in solid-state cultures. Afr. J. Biotechnol. 2017, 16, 672-682.

106. Chadni, Z.; Rahaman, M.H.; Jerin, I.; Hoque, K.M.F.; Reza, M.A. Extraction and optimization of red pigment production as secondary metabolites from Talaromyces verruculosus and its potential use in textile industries. Mycology 2017, 8, 48-57. [CrossRef]

107. Koolen, H.H.F.; Menezes, L.S.; Souza, M.P.; Silva, F.M.A.; Almeida, F.G.O.; de Souza, A.Q.L.; Nepel, A.; Barison, A.; da Silva, F.H.; Evangelistae, D.E.; et al. Talaroxanthone, a novel xanthone dimer from the endophytic fungus Talaromyces sp. associated with Duguetia stelechantha (Diels) R. E. Fries. J. Braz. Chem. Soc. 2013, 24, 880-883.

108. Morales-Oyervides, L.; Oliveira, J.; Sousa-Gallagher, M.; Mendez-Zavala, A.; Montanez, J.C. Assessment of the dyeing properties of the pigments produced by Talaromyces spp. J. Fungi 2017, 3, 38. [CrossRef]

109. Zhai, M.-M.; Li, J.; Jiang, C.-X.; Shi, Y.P.; Di, D.L.; Crews, P.; Wu, Q.-X. The bioactive secondary metabolites from Talaromyces species. Nat. Prod. Bioprospect. 2016, 6, 1-24. [CrossRef]

110. Pattenden, G. Synthesis of Asperenone, a new pigment from Aspergillus niger and Aspergillus awamori. Tetrahedron Lett. 1969, 10, 4049-4052. [CrossRef]

111. Youngchim, S.; Morris-Jones, R.; Hay, R.J.; Hamilton, A.J. Production of melanin by Aspergillus fumigatus. J. Med. Microbiol. 2004, 53, 175-181. [CrossRef] [PubMed]

112. Brown, D.W.; Solvo, J.J. Isolation and characterization of sexual spore pigments from Aspergillus nidulans. Appl. Environ. Microbiol. 1994, 60, 979-983. [PubMed]

113. Wang, C.C.; Chiang, Y.M.; Kuo, P.L.; Chang, J.K.; Hsu, Y.L. Norsolorinic acid from Aspergillus nidulans inhibits the proliferation of human breast adenocarcinoma MCF-7 cells via Fas-mediated pathway. Basic Clin. Pharmacol. Toxicol. 2008, 102, 491-497. [CrossRef] [PubMed]

114. Ray, A.C.; Eakin, R.E. Studies on the biosynthesis of Aspergillin by Aspergillus niger. J. Appl. Microbiol. 1975, 30, 909-915.

115. Zabala, A.O.; Xu, W.; Chooi, Y.-H.; Tang, Y. Discovery and characterization of a silent gene cluster that produces azaphilones from Aspergillus niger ATCC 1015 reveal a hydroxylation-mediated pyran-ring formation. Chem. Biol. 2012, 19, 1049-1059. [CrossRef] [PubMed]

116. Hosoe, T.; Mori, N.; Kamano, K.; Itabashi, T.; Yaguchi, T.; Kawai, K. A new antifungal yellow pigment from Aspergillus nishimurae. J. Antibiot. 2011, 64, 211-212. [CrossRef] [PubMed]

117. Li, D.-L.; Li, X.-M.; Wang, B.-G. Natural anthraquinone derivatives from a marine mangrove plant-derived endophytic fungus Eurotium rubrum: Structural elucidation and DPPH radical scavenging activity. J. Biotechnol. 2009, 19, 675-680.

118. Akilandeswari, P.; Pradeep, B.V. Aspergillus terreus KMBF1501 a potential pigment producer under submerged fermentation. Int. J. Pharm. Pharm. Sci. 2017, 9, 38-43. [CrossRef]

119. Assante, G.; Camarda, L.; Locci, R.; Merlini, L. Isolation and structure of red pigments from Aspergillus flavus and related species, grown on a differential medium. J. Agric. Food Chem. 1981, 29, 785-787. [CrossRef]

120. Narendrababu, B.N.; Shishupala, S. Spectrophotometric detection of pigments from Aspergillus and Penicillium isolates. J. Appl. Biol. Biotechnol. 2017, 5, 53-58. [CrossRef] 
121. Cambaza, E. Comprehensive description of Fusarium graminearum pigments and related compounds. Foods 2018, 7, 165. [CrossRef] [PubMed]

122. Wollenberg, R.D.; Saei, W.; Westphal, K.R.; Klitgaard, C.S.; Nielsen, K.L.; Lysøe, E.; Gardiner, D.M.; Wimmer, R.; Sondergaard, T.S.; Sørensen, J.L. Chrysogine biosynthesis is mediated by a two-module nonribosomal peptide synthetase. J. Nat. Prod. 2017, 80, 2131-2135. [CrossRef] [PubMed]

123. Sardaryan, E. Strain of the Microorganism Penicillium oxalicum var. Armeniaca and Its. Application. Patent EP1070136B1, 4 August 2004.

124. Gupta, C.; Sharma, D.; Aggarwal, S.; Nagpal, N. Pigment production from Trichoderma spp. for dyeing of silk and wool. Int. J. Sci. Nat. 2013, 4, 351-355.

125. Takahashi, S.; Uchida, K.; Kakinuma, N.; Hashimoto, R.; Yanagisawa, T.; Nakagawa, A. The structures of Pyridovericin and Pyridomacrolidin, new metabolites from the entomopathogenic fungus, Beauveria bassiana. J. Antibiot. 1998, 51, 1051-1054. [CrossRef] [PubMed]

126. Cho, Y.J.; Park, J.P.; Hwang, H.J.; Kim, S.W.; Choi, J.W.; Yun, J.W. Production of red pigment by submerged culture of Paecilomyces sinclairii. Lett. Appl. Microbiol. 2002, 35, 195-202. [CrossRef] [PubMed]

127. Kot, A.M.; Błażejak, S.; Gientka, I.; Kieliszek, M.; Bryś, J. Torulene and torularhodin: "new" fungal carotenoids for industry? Microb. Cell Fact. 2018, 17, 49. [CrossRef]

128. Pollmann, H.; Breitenbach, J.; Wolff, H.; Bode, H.B.; Sandmann, G. Combinatorial biosynthesis of novel multi-hydroxy carotenoids in the red yeast Xanthophyllomyces dendrorhous. J. Fungi 2017, 3, 9. [CrossRef]

129. Fouillaud, M.; Venkatachalam, M.; Girard-Valenciennes, E.; Caro, Y.; Dufossé, L. Anthraquinones and derivatives from marine-derived fungi: Structural diversity and selected biological activities. Mar. Drugs 2016, 14, 64. [CrossRef]

130. Fouillaud, M.; Venkatachalam, M.; Llorente, M.; Magalon, H.; Cuet, P.; Dufossé, L. Biodiversity of pigmented fungi isolated from the marine environment in La Reunion Island, Indian Ocean: New resources for colored metabolites. J. Fungi 2017, 3, 36. [CrossRef]

131. Venkatachalam, M.; Zelena, M.; Cacciola, F.; Ceslova, L.; Girard-Valenciennes, E.; Clerc, P.; Dugo, P.; Mondello, L.; Fouillaud, M.; Rotondo, A.; et al. Partial characterization of the pigments produced by the marine-derived fungus Talaromyces albobiverticillius 30548. Towards a new fungal red colorant for the food industry. J. Food Compost. Anal. 2018, 67, 38-47. [CrossRef]

132. Tseng, Y.Y.; Chen, M.T.; Lin, C.F. Growth, pigment production and protease activity of Monascus purpureus as affected by salt, sodium nitrite, polyphosphate, and various sugars. J. Appl. Microbiol. 2000, 88, 3-37. [CrossRef] [PubMed]

133. Chatterjee, S.; Maity, S.; Chattopadhyay, P.; Sarkar, A.; Laskar, S.; Sen, S.K. Characterization of red pigment from Monascus in submerged cultured pigment from Monascus purpureus. J. Appl. Sci. Res. 2009, 5, 2102-2108.

134. Carvalho, J.C.; Oishi, B.O.; Woiciechowski, A.L.; Pandey, A.; Babitha, S.; Soccol, C.R. Effect of substrates on the production of Monascus biopigments by solid-state fermentation and pigment extraction using different solvents. Indian J. Biotechnol. 2007, 6, 194-199.

135. Patil, S.A.; Sivanandhan, G.; Thakare, D.B. Effect of physical and chemical parameters on the production of red exopigment from Penicillium purpurogenum isolated from spoilt onion and study of its antimicrobial activity. Int. J. Curr. Microbiol. Appl. Sci. 2015, 4, 599-609.

136. Lopes, F.C.; Tichota, D.M.; Pereira, J.Q.; Segalin, J.; Rios, A.D.O. Pigment production by filamentous fungi on agro-industrial byproducts: An eco-friendly alternative. Appl. Biochem. Biotechnol. 2013, 171, 616-625. [CrossRef]

137. Srianta, I.; Zubaidah, E.; Estiasih, T.; Yamada, M. Comparison of Monascus purpureus growth, pigment production and composition on different cereal substrates with solid state fermentation. Biocatal. Agric. Biotechnol. 2016, 7, 181-186. [CrossRef]

138. Kantifedaki, A.; Kachrimanidou, V.; Mallouchos, A.; Papanikolaou, S.; Koutinas, A.A. Orange processing waste valorisation for the production of bio-based pigments using the fungal strains Monascus purpureus and Penicillium purpurogenum. J. Clean. Prod. 2018, 185, 882-890. [CrossRef]

139. Costa, J.P.V.; Vendruscolo, F. Production of red pigments by Monascus ruber CCT 3802 using lactose as a substrate. Biocatal. Agric. Biotechnol. 2017, 11, 50-55. [CrossRef]

140. Chen, M.; Johns, M.R. Effect of $\mathrm{pH}$ and nitrogen source on pigment production by Monascus purpureus. Appl. Microbiol. Biotechnol. 1993, 40, 132-138. [CrossRef] 
141. Blanc, P.J.; Loret, M.O.; Santerre, A.L.; Pareilleux, A.; Prome, D.; Prome, J.C.; Laussac, J.P.; Goma, G. Pigments of Monascus. J. Food Sci. 1994, 59, 862-865. [CrossRef]

142. Pastrana, L.; Blanc, P.J.; Santerre, A.L.; Loret, M.O.; Goma, G. Production of red pigments by Monascus ruber in synthetic media with a strictly controlled nitrogen source. Process Biochem. 1995, 30, 333-341. [CrossRef]

143. Zhang, X.; Wang, J.; Chen, M.; Wang, C. Effect of nitrogen sources on production and photostability of Monascus pigments in liquid fermentation. IERI Procedia 2013, 5, 344-350. [CrossRef]

144. Zhen, Z.; Xiong, X.; Liu, Y.; Zhang, J.; Wang, S.; Li, L.; Gao, M. NaCl inhibits citrinin and stimulates Monascus pigments and monacolin K production. Toxins 2019, 11, 118. [CrossRef]

145. Stange, S.; Steudler, S.; Delenk, H.; Werner, A.; Walther, T.; Wagenführ, A. Influence of the nutrients on the biomass and pigment production of Chlorociboria aeruginascens. J. Fumgi 2019, 5, 40. [CrossRef]

146. Carels, M.; Shepherd, D. The effect of $\mathrm{pH}$ and amino acids on conidiation and pigment production of Monascus major ATCC 16362 and Monascus rubiginosus ATCC 16367 in submerged shaken culture. Can. J. Microbiol. 1978, 24, 1346-1357. [CrossRef]

147. Jung, H.; Kim, C.; Kim, K.; Shin, C.S. Color characteristics of Monascus pigments derived by fermentation with various amino acids. J. Agric. Food Chem. 2003, 51, 1302-1306. [CrossRef]

148. Li, L.; Chen, S.; Gao, M.; Ding, B.; Zhang, J.; Zhou, Y.; Liu, Y.; Yang, H.; Wu, Q.; Chen, F. Acidic conditions induce the accumulation of orange Monascus pigments during liquid-state fermentation of Monascus ruber M7. Appl. Microbio. Biotech. 2019, 103, 8393-8402. [CrossRef]

149. Mawthols, K.R.; Deshpande, R.; Ware, D.; Mahajan, M. Effect of $\mathrm{pH}$ on pigment production of fungi and their toxicity on seed germination. Ecol. Environ. Conserv. 2005, 11, 325-326.

150. Mendez, A.; Perez, C.; Montanez, J.C.; Martinez, G.; Aguilar, C.N. Red pigment production by Penicillium purpurogenum $\mathrm{GH} 2$ is influenced by $\mathrm{pH}$ and temperature. J. Zhejiang Univ. Sci. B (Biomed. Biotechnol.) 2011, 12, 961-968. [CrossRef]

151. Afsharia, M.; Shahidia, F.; Mortazavia, S.A.; Tabatabaia, F.; Es'haghib, Z. Investigating the influence of $\mathrm{pH}$, temperature and agitation speed on yellow pigment production by Penicillium aculeatum ATCC 10409. Nat. Prod. Res. 2015, 29, 1300-1306. [CrossRef] [PubMed]

152. Stange, S.; Steudler, S.; Delenk, H.; Werner, A.; Walther, T.; Wagenführ, A. Influence of environmental growth factors on the biomass and pigment production of Chlorociboria aeruginascens. J. Fungi 2019, 5, 46. [CrossRef] [PubMed]

153. Huang, T.; Tan, H.; Chen, G.; Wang, L.; Wu, Z. Rising temperature stimulates the biosynthesis of water-soluble fluorescent yellow pigments and gene expression in Monascus ruber CGMCC10910. AMB Express 2017, 7, 134. [CrossRef] [PubMed]

154. Zhou, B.; Tian, Y.; Zhong, H. Application of a two-stage agitation speed control strategy to enhance yellow pigments production by Monascus anka mutant. J. Microbiol. Biotechnol. Food Sci. 2019, 8, 1260-1264. [CrossRef]

155. Velmurugan, P.; Lee, Y.H.; Venil, C.K.; Lakshmanaperumalsamy, P.; Chae, J.C.; Oh, B.T. Effect of light on growth, intracellular and extracellular pigment production by five pigment-producing filamentous fungi in synthetic medium. J. Biosci. Bioeng. 2010, 109, 346-350. [CrossRef] [PubMed]

156. Buhler, R.M.M.; Muller, B.L.; Moritz, D.E.; Vendruscolo, F.; Oliveira, D.; Ninow, J.L. Influence of light intensity on growth and pigment production by Monascus ruber in submerged fermentation. Appl. Biochem. Biotechnol. 2015, 176, 1277-1289. [CrossRef]

157. Haggblom, P.; Unestam, T. Blue light inhibits mycotoxin production and increases total lipids and pigmentation in Alternaria alternata. Appl. Environ. Microbiol. 1979, 38, 1074-1077.

158. Palacio-Barrera, A.M.; Areiza, D.; Zapata, P.; Atehortúa, L.; Correa, C.; Peñuela-Vásquez, M. Induction of pigment production through media composition, abiotic and biotic factors in two filamentous fungi. Biotechnol. Rep. 2019, 21, e00308. [CrossRef]

159. Tudor, D.; Robinson, S.C.; Cooper, P.A. The influence of moisture content variation on fungal pigment formation in spalted wood. AMB Express 2012, 2, 69. [CrossRef]

160. Gunsekaran, S.; Poorniammal, R. Optimization of fermentation conditions for red pigment production from Penicillium sp. under submerged cultivation. Afr. J. Biotechnol. 2008, 7, 1894-1898. [CrossRef]

161. Chutia, M.; Ahmed, G.U. Optimization of biomass and pigment production by Penicillium species isolated from virgin forest floor. Biotechnol. 2012, 6, 61-69. 
162. Pradeep, F.S.; Pradeep, B.V. Optimization of pigment and biomass production from Fusarium moniliforme under submerged fermentation conditions. Int. J. Pharm. Pharm. Sci. 2013, 5, 526-535.

163. Ahmad, M.; Panda, B.P. Optimization of red pigment production by Monascus purpureus MTCC 369 under solid-state fermentation using response surface methodology. Songklanakarin J. Sci. Technol. 2014, 36, 439-444.

164. Devi, S.; Karuppan, P. Influence of culture condition and $\mathrm{pH}$ on growth and production of brown pigment from Alternaria alternata. Int. J. Sci. Res. 2014, 3, 458-461.

165. Santos-Ebinuma, V.C.; Roberto, I.C.; Teixeira, M.F.S.; Pessoa, J., Jr. Improvement of submerged culture conditions to produce colorants by Penicillium purpurogenum. Braz. J. Microbiol. 2014, 45, 731-742. [CrossRef] [PubMed]

166. Seyedin, A.; Yazdian, F.; Hatamian-Zarmi, A.; Rasekh, B.; Mir-derikvand, M. Natural pigment production by Monascus purpureus: Bioreactor yield improvement through statistical analysis. Appl. Food Biotechnol. 2015, 2, 23-30.

167. Patrovsky, M.; Sinovska, K.; Branska, B.; Patakova, P. Effect of initial pH, different nitrogen sources, and cultivation time on the production of yellow or orange Monascus purpureus pigments and the mycotoxin citrinin. Food Sci. Nutr. 2019, 1-7. [CrossRef]

168. Serrano, R.; González-Menéndez, V.; Rodríguez, L.; Martín, J.; Tormo, J.R.; Genilloud, O. Co-culturing of fungal strains against Botrytis cinerea as a model for the induction of chemical diversity and therapeutic agents. Front. Microbial. 2017, 8, 649. [CrossRef]

169. Tan, Z.Q.; Leow, H.Y.; Lee, D.C.W.; Karisnan, K.; Song, A.A.L.; Mai, C.W.; Yap, W.S.; Lim, S.H.E.; Lai, K.S. Co-culture Systems for the production of secondary metabolites: Current and future prospects. Open Biotechnol. J. 2019, 13, 18-26. [CrossRef]

170. Shin, C.S.; Kim, H.J.; Kim, M.J.; Ju, J.Y. Morphological change and enhanced pigment production of Monascus when cocultured with Saccharomyces cerevisiae or Aspergillus oryzae. Biotechnol. Bioeng. 1998, 59, 576-581. [CrossRef]

171. Frases, S.; Chaskes, S.; Dadachova, E.; Casadevall, A. Induction by Klebsiella aerogenes of a melanin-like pigment in Cryptococcus neoformans. Appl. Environ. Microbiol. 2006, 72, 1542-1550. [CrossRef] [PubMed]

172. Smith, K.T. Zone lines. In Encyclopedia of Plant Pathology; Malloy, O.C., Murray, T.D., Eds.; John Wiley \& Sons: Hoboken, NJ, USA, 2001; Volume 2, pp. 1217-1218.

173. Robinson, S. The fine art of decay. Am. Sci. 2014, 102, 206-213. [CrossRef]

174. Robinson, S.C. Developing fungal pigments for "painting" vascular plants. Appl. Microbiol. Biotechnol. 2012, 93, 1389-1394. [CrossRef] [PubMed]

175. Robinson, S.C.; Hinsc, E.; Weber, G.L.; Freitas, S. Method of extraction and resolubilisation of pigments from Chlorociboria aeruginosa and Scytalidium cuboideum, two prolific spalting fungi. Coloration Technol. 2014, 130, 221-225. [CrossRef]

176. Morales-Oyervides, L.; Oliveira, J.; Sousa-Gallagher, M.; Mendez-Zavala, A.; Montanez, J.C. Perstraction of intracellular pigments through submerged fermentation of Talaromyces spp. in a surfactant-rich media: A novel approach for enhanced pigment recovery. J. Fungi 2017, 3, 33. [CrossRef]

177. Kaur, S.; Panesar, P.S.; Gurumayum, S.; Rasane, P.; Kumar, V. Optimization of aqueous extraction of orevactaene and flavanoid pigments produced by Epicoccum nigrum. Pigment Resin Technol. 2019, 48, 301-308. [CrossRef]

178. Weber, G.L.; Boonloed, A.; Naas, K.M.; Koesdjojo, M.T.; Remcho, V.T.; Robinson, S.C. A method to stimulate production of extracellular pigments from wood-degrading fungi using a water carrier. Curr. Res. Environ. Appl. Mycol. 2016, 6, 218-230. [CrossRef]

179. Sen, T.; Barrow, C.J.; Deshmukh, S.K. Microbial pigments in the food industry-Challenges and the way forward. Front. Nutr. 2019, 6, 7. [CrossRef]

180. Jones, J.D.; Hohn, T.M.; Leathers, T.D. Genetically modifed strains of Fusarium sporotrichioides for production of lycopene and $\beta$-carotene. In Proceedings of the Society of Industrial Microbiology Annual Meeting, San Diego, CA, USA, 29 July 2004; p. 91.

181. Fu, G.; Xu, Y.; Li, Y.; Tan, W. Construction of a replacement vector to disrupt pksCT gene for the mycotoxin citrinin biosynthesis in Monascus aurantiacus and maintain food red pigment production. Asia Pacif. J. Clin. Nutr. 2007, 16, 137-142.

182. Jia, X.Q.; Xu, Z.N.; Zhou, L.P.; Sung, C.K. Elimination of the mycotoxin citrinin production in the industrial important strain Monascus purpureus SM001. Metab. Eng. 2010, 12, 1-7. [CrossRef] 
183. Westphal, K.; Wollenberg, R.; Herbst, F.A.; Sørensen, J.; Sondergaard, T.; Wimmer, R. Enhancing the production of the fungal pigment aurofusarin in Fusarium graminearum. Toxins 2018, 10, 485. [CrossRef] [PubMed]

184. Guo, X.; Li, Y.; Zhang, R.; Yu, J.; Ma, X.; Chen, M.; Wang, Y. Transcriptional regulation contributes more to Monascus pigments diversity in different strains than to DNA sequence variation. World J. Microbiol. Biotechnol. 2019, 35, 138. [CrossRef] [PubMed]

185. Dufossé, L.; Galaup, P.; Yaron, A.; Arad, S.M.; Blanc, P.; Murthy, K.N.C.; Ravishankar, G.A. Microorganisms and microalgae as sources of pigments for food use: A scientific oddity or an industrial reality? Trends Food Sci. Technol. 2005, 16, 389-406. [CrossRef]

186. Mapari, S.A.S.; Meyer, A.S.; Thrane, U. Colorimetric characterization for comparative analysis of fungal pigments and natural food colorants. J. Agric. Food Chem. 2006, 54, 7027-7035. [CrossRef]

187. Simpson, B.K.; Benjakul, S.; Klomklao, S. Chapter 37. Natural Food Pigments. In Food Biochemistry and Food Processing, 2nd ed.; Simpson, B.K., Ed.; Wiley-Blackwell: Hoboken, NJ, USA, 2012; pp. 704-722.

188. Fink-Gremmels, J.; Leistner, L. Biologische Wirkungen von Monascus purpureus. Fleischwutsch 1989, 69, 116-122.

189. Kim, D.; Ku, S. Beneficial effects of Monascus sp. KCCM 10093 pigments and derivatives: A mini review. Molecules 2018, 23, 98. [CrossRef]

190. Martinkova, L.; Juzlova, P.; Vesely, D. Biological activity of polyketide pigments produced by the fungus Monascus. J. Appl. Bacteriol. 1995, 79, 609-616. [CrossRef]

191. Vendruscolo, F.; Tosin, I.; Giachini, A.J.; Schmidell, W.; Ninow, J.L. Antimicrobial activity of Monascus pigments produced in submerged fermentation. J. Food Process. Preserv. 2014, 38, 1860-1865. [CrossRef]

192. Manon Mani, V.; Shanmuga Priya, M.; Dhaylini, S.; Preethi, K. Antioxidant and antimicrobial evaluation of bioactive pigment from Fusarium sp. isolated from the stressed environment. Int. J. Curr. Microbiol. Appl. Sci. 2015, 4, 1147-1158.

193. Saravanan, D.; Radhakrishnan, M. Antimicrobial activity of pigments produced by fungi from the Western Ghats. J. Chem. Pharm. Res. 2016, 8, 634-638.

194. Yolmeh, M.; Hamedi, H.; Khomeiri, M. Antimicrobial activity of pigments extracted from Rhodotorula glutinis against some bacteria and fungi. Zahedan J. Res. Med Sci. 2016, 18, e4954. [CrossRef]

195. Poorniammal, R.; Parthiban, M.; Gunasekaran, S.; Murugesan, R.; Thilagavathi, G. Natural dye production from Thermomyces sp. fungi for textile application. Indian J. Fibre Text. Res. 2013, 38, 276-279.

196. Devi, S.; Karuppan, P. Reddish brown pigments from Alternaria alternata for textile dyeing and printing. Indian J. Fibre Text. Res. 2015, 40, 315-319.

197. Prathiban, M.; Thilagavathi, G.; Viju, S. Development of antibacterial silk sutures using the natural fungal extract for healthcare applications. J. Text. Sci. Eng. 2016, 6, 249.

198. Tuli, H.S.; Chaudhary, P.; Beniwal, V.; Sharma, A.K. Microbial pigments as natural color sources: Current trends and future perspectives. J. Food Sci. Technol. 2015, 52, 4669-4678. [CrossRef] [PubMed]

199. Vendruscolo, F.; Buhler, R.M.M.; de Carvalho, J.C.; de Oliveira, D.; Moritz, D.E.; Schmidell, W.; Ninow, J.L. Monascus: A reality on the production and application of microbial pigments. J. Appl. Biochem. Biotechnol. 2016, 178, 211-223. [CrossRef]

200. Li, F.; Xue, F.; Yu, X. GC-MS, FTIR and Raman analysis of antioxidant components of red pigments from Stemphylium lycopersici. Curr. Microbiol. 2017, 74, 532-539. [CrossRef]

201. Poorniammal, R.; Prabhu, S.; Sakthi, A.R. Evaluation of in vitro antioxidant activity of fungal pigments. Pharma Innov. J. 2019, 8, 326-330.

202. Malik, K.; Tokas, J.; Anand, R.C. Characterization and cytotoxicity assay of pigment-producing microbes. Int. J. Curr. Microbiol. Appl. Sci. 2016, 5, 370-376. [CrossRef]

203. Poorniammal, R.; Prabhu, S.; Sakthi, A.R.; Gunasekaran, S. Subacute dermal toxicity of Thermomyces sp. and Penicillium purpurogenum pigments in wistar rats. Int. J. Chem. Stud. 2019, 7, 630-634.

204. Akihisa, T.; Tokuda, H.; Ukiya, M.; Kiyota, A.; Yasukawa, K.; Sakamoto, N.; Kimura, Y.; Suzuki, T.; Takayasu, J.; Nishino, H. Anti-tumor-initiating effects of Monascin, an azaphilonoid pigment from the extract of Monascus pilosus fermented rice (red-mold rice). Chem. Biodivers. 2005, 2, 1305-1309. [CrossRef] [PubMed]

205. Su, N.-W.; Lin, Y.-L.; Lee, M.-H.; Ho, C.-Y. Ankaflavin from Monascus-fermented red rice exhibits selective cytotoxic effect and induces cell death on Hep G2 cells. J. Agric. Food Chem. 2005, 53, 1949-1954. [CrossRef] [PubMed] 
206. Hill, H. The function of melanin or six blind people examine an elephant. BioEssays 1992, 14, 49-56. [CrossRef] [PubMed]

207. Sajid, S.; Akber, N. Applications of fungal pigments in biotechnology. Pure Appl. Biol. 2018, 7, 922-930. [CrossRef]

208. Velmurugan, P.; Kim, M.J.; Park, J.S.; Karthikeyan, K.; Lakshmanaperumalsamy, P.; Lee, K.J.; Park, Y.J.; Oh, B.T. Dyeing of cotton yarn with five water-soluble fungal pigments obtained from five fungi. Fibers Polym. 2010, 11, 598-605. [CrossRef]

209. Mabrouk, A.M.; El-Kkhrisy, E.A.M.; Youssef, Y.A.; Asem, M.A. Production of textile reddish brown dyes by fungi. Malays. J. Microbiol. 2011, 7, 33-40.

210. Sharma, D.; Gupta, C.; Aggarwal, S.; Nagpal, N. Pigment extraction from fungus for textile dyeing. Indian J. Fibre Text. Res. 2012, 37, 68-73.

211. Aishwarya, A.D. Extraction of natural dyes from fungus-An alternate for textile dyeing. J. Nat. Sci. Res. 2014, 4, 1-6.

212. Weber, G.; Chen, H.L.; Hinsch, E.; Freitas, S.; Robinson, S. Pigments extracted from the wood-staining fungi Chlorociboria aeruginosa, Scytalidium cuboideum, and S. ganodermophthorum show potential for use as textile dyes. Coloration Technol. 2014, 130, 445-452. [CrossRef]

213. Robinson, S.C.; Tudor, D.; Cooper, P.A. Utilizing pigment-producing fungi to add commercial value to American beech (Fagus grandifolia). Appl. Microbial. Biotechnol. 2012, 93, 1041-1048. [CrossRef]

214. Giesbers, G.; Van Schenck, J.; Gutierrez, S.V.; Robinson, S.; Ostroverkhova, O. Fungi-derived pigments for sustainable organic (opto) electronics. MRS Adv. 2018, 3, 3459-3464. [CrossRef]

215. Giesbers, G.; Van Schenck, J.; Quinn, A.; Van Court, R.; Vega Gutierrez, S.M.; Robinson, S.C.; Ostroverkhova, O. Xylindein: Naturally produced fungal compound for sustainable (opto) electronics. ACS Omega 2019, 4, 13309-13318. [CrossRef] [PubMed]

(C) 2019 by the authors. Licensee MDPI, Basel, Switzerland. This article is an open access article distributed under the terms and conditions of the Creative Commons Attribution (CC BY) license (http://creativecommons.org/licenses/by/4.0/). 